\title{
Experimental modal analysis of nonlinear systems by using response-controlled stepped-sine testing
}

\author{
Taylan Karaağaçlı ${ }^{\mathrm{a}}$, H. Nevzat Özgüven ${ }^{\mathrm{b}, *}$ \\ ${ }^{a}$ The Scientific and Technological Research Council of Turkey, Defense Industries Research and Development Institute, TÜBITAK-SAGE, P.K. 16, 06261 \\ Mamak, Ankara, Turkey \\ ${ }^{\mathrm{b}}$ Mechanical Engineering Department, Middle East Technical University, 06800 Ankara, Turkey
}

\section{A R T I C L E I N F O}

\section{Article history:}

Received 23 December 2019

Received in revised form 25 May 2020

Accepted 30 May 2020

Available online 11 June 2020

\section{Keywords:}

Nonlinear experimental modal analysis

Nonlinear system identification

Response controlled stepped sine test

Nonlinear mode

Harmonic force surface

Unstable branch

\begin{abstract}
A B S T R A C T
Although the identification and analysis of structures with a localized nonlinearity, either weak or strong, is within reach, identification of multiple nonlinearities coexisting at different locations is still a challenge, especially if these nonlinearities are strong. In such cases, identifying each nonlinearity separately requires a tedious work or may not be possible at all in some cases. In this paper, an approach for experimental modal analysis of nonlinear systems by using Response-Controlled stepped-sine Testing (RCT) is proposed. The proposed approach is applicable to systems with several nonlinearities at various different locations, provided that modes are well separated and no internal resonances occur. Step-sine testing carried out by keeping the displacement amplitude of the driving point constant yields quasi-linear frequency response functions directly, from which the modal parameters can be identified as functions of modal amplitude of the mode of concern, by employing standard linear modal analysis tools. These identified modal parameters can then be used in calculating near-resonant frequency response curves, including the unstable branch if there is any, for various untested harmonic forcing cases. The proposed RCT approach makes it also possible to extract nonlinear normal modes experimentally without using sophisticated control algorithms, directly from the identified modal constants, and also to obtain near-resonant frequency response curves experimentally for untested constant-amplitude harmonic forcing cases by extracting isocurves of constantamplitude forcing from the measured Harmonic Force Surface (HFS), a new concept proposed in this paper. The key feature of the HFS is its ability to extract unstable branches together with turning points of constant-force frequency response curves directly from experiment, accurately. The method is validated with numerical and experimental case studies. The numerical example consists of a 5 DOF lumped system with strong several conservative nonlinear elements. Experimental case studies consist of a cantilever beam supported at its free-end by two metal strips which create strong stiffening nonlinearity, and a real missile structure which exhibit moderate damping nonlinearity mostly due to several bolted joints on the structure.
\end{abstract}

(c) 2020 Elsevier Ltd. All rights reserved.

\footnotetext{
* Corresponding author.

E-mail address: ozguven@metu.edu.tr (H.N. Özgüven).
} 


\section{Introduction}

Structural dynamics community witnessed important developments in the field of nonlinear system identification over the last two decades [1,2]. Despite the significant progress in the state-of-the-art, there are still difficult problems such as joint nonlinearities (multiple and discrete) or geometric (i.e. continuously distributed) nonlinearities due to large deformations. Since there are generally several joints in engineering structures, it would be very difficult, if not impossible, to identify each joint nonlinearity separately. On the other hand, in case of geometric nonlinearities, the concept of discrete nonlinear elements cannot be used. Consequently, the right identification strategy in these complex problems is to quantify the resultant effect of all nonlinearities, instead of focusing on individual nonlinear elements. Fortunately, the concept of nonlinear normal modes (NNMs), which dates back to Rosenberg [3,4] (1960s), provides a rigorous theoretical framework to study the overall effect of several nonlinearities in a structure. Analogous to linear normal modes, Rosenberg defined an NNM as a vibration in unison of the system, i.e. synchronous periodic motion. Later, in 1979, Szemplinska-Stupnicka proposed a novel technique called the single nonlinear mode method [5] to study the effect of nonlinearities on the resonant vibrations of multi-degree-of-freedom (MDOF) systems. The method showed that if the modes are well separated and no pronounced modal coupling occurs in the energy range of interest, near-resonant frequency responses of a nonlinear system can be represented accurately by a single NNM and its corresponding natural frequency which are functions of modal amplitude. This pioneering work of Szemplinska-Stupnicka led to the development of various nonlinear modal identification techniques. For example, in the early 1990s, Setio et al. [6] proposed to extract nonlinear modal parameters by minimizing the error function between frequency responses measured by force-controlled sine-sweep testing and analytical frequency responses represented by superposition of NNMs, which gives satisfactory results if the modes are well separated and no internal resonances occur. The method was validated on a real cantilever beam supported at its free end by a string which creates cubic stiffness effect. Later, in the early 2000s, Gibert [7] proposed a similar modal identification algorithm based on minimization of the error function between measured and analytical frequency responses and showed that superposition of NNMs gives satisfactory results for the synthesis of frequency responses of the Ecole-Central-de-Lyon (ECL) benchmark [8] over the frequency range including the first three elastic modes.

Early 2000s witnessed other promising nonlinear modal identification strategies. For example, Göge et al. [9] proposed a novel method called the identification of nonlinearity by time-series-based linearity plots (INTL) which is based on the application of the restoring force surface (RFS) method [10] in modal space. The test strategy used in the INTL approach is the normal mode force appropriation which is also known as phase resonance testing, where the nonlinear system is harmonically excited at its resonance frequency by means of an excitation force pattern appropriated to a single mode of interest. Nonlinear modal stiffness and damping parameters can then be determined by curve fitting to the measured nonlinear restoring force in modal space. The application of the method was demonstrated on a real space structure. Platten et al. [11] also proposed another RFS-based technique called the nonlinear resonant decay method (NLRDM). This method differs from the INTL approach in that it can take also the cross-coupling of nonlinear modes into account by virtue of its excitation strategy which consists of a burst sine at the resonance frequency of the mode of interest. The NLRDM technique was validated on a wing-like structure with hardening stiffness at the pylon connections [12] and on a real transport aircraft [13].

The major difference between the new generation techniques developed in the last decade and the afore-mentioned ones is that in the new generation algorithms, computational effort is minimized at the cost of experimental effort. Most of the recently developed nonlinear modal identification methods are inspired by phase resonance testing approach and they focus on direct parameter estimation, which reduces computational effort considerably. For example, in the method proposed by Peeters et al. [14] in 2011, the phase lag quadrature criterion was generalized to nonlinear structures in order to locate a single NNM during experiment. Once the NNM appropriation is achieved, the frequency-energy dependence of that nonlinear mode can be determined by applying time-frequency analysis to the free decay response data. The proposed methodology was demonstrated on numerical examples [14] and on a real nonlinear beam structure [15]. However, an important drawback of this early version of the nonlinear phase resonance testing was the manual tuning process of the phase lag between response and excitation. By virtue of the recently proposed control algorithms [16,17], the tuning of the phase lag was automated throughout the entire NNM backbone curve. The control technique proposed by Peter and Leine in [16] is called phase-locked-loop (PLL) control which provides a robust and fast way of tracing out back-bone curves as well as of stabilizing the unstable branches of near-resonant frequency response curves. The PLL control strategy, which outputs zeroth and higher harmonics of nonlinear modes as well as the fundamental harmonic, was validated on a benchmark beam structure in [16] and on a circular plate, a chinese gong and a piezoelectric cantilever beam in [18]. In accordance with the single nonlinear mode assumption [5], synthesis of the near-resonant frequency response curve of a nonlinear cantilever beam from a single NNM measured by PLL technique was also demonstrated in [19]. Another interesting application of the PLL technique is the identification of the nonlinear dissipation at a bolted joint [20]. The control approach proposed by Renson et al. in [17], called control-based continuation (CBC), is similar to the PLL method, and also enables backbone curve identification of nonlinear structures. The application of the $\mathrm{CBC}$ method was demonstrated on a real singledegree-of-freedom (SDOF) oscillator in [17].

All of the nonlinear modal identification techniques mentioned above have their own advantages and limitations. Early identification methods [6,7] which rely on simple frequency response data measured by standard force-controlled sine sweep testing involve considerable computational cost. On the other hand, INLT and NLRDM require considerable effort both 
in computation and experiment, and they are applicable to weakly nonlinear systems. In nonlinear phase resonance testing method [14], the computational effort is considerable reduced, but the manual tuning of the phase lag between response and excitation process requires careful and time consuming experimentation. Although recently developed PLL and CBC control strategies automated the tuning of the phase lag as well as the determination of the backbone curve, they cannot make use of the available standard equipment. Furthermore, although experimental extraction of natural frequencies and deflection shapes at resonance is straightforward in the methods based on phase resonance testing approach, determination of nonlinear modal damping is still an important issue.

In this paper, an approach for experimental modal analysis of nonlinear systems by using response-controlled stepped-sine testing (RCT) is proposed. The proposed approach is applicable to systems with several nonlinearities at various different locations, provided that modes are well separated and no internal resonances occur. The method is based on the single nonlinear mode assumption of Szemplinska-Stupnicka [5], where near-resonant frequency responses of a nonlinear system can be represented accurately by a single NNM and its corresponding natural frequency which are functions of a single modal amplitude. Accordingly, the proposed method hypothesizes that if the displacement (equivalently modal) amplitude is kept constant with the RCT strategy during modal testing, measured frequency response functions (FRFs) come out in quasi-linear form. That makes it possible to use standard linear modal analysis tools to extract all modal parameters as functions of modal amplitude. These identified modal parameters can then be employed to synthesize near-resonant frequency response curves including unstable branches, if there is any, for various untested harmonic forcing scenarios. Furthermore, in case of using multiple sensors during RCT, NNMs can also be experimentally extracted from identified modal constants. Therefore, the contribution of the proposed method is threefold. Firstly, it relies on standard controllers (available in commercial modal testing hardware and driven by commercial software) which makes it very attractive especially for industrial applications. Secondly, identification of modal damping and mass normalization of NNMs, which are necessary for the prediction of frequency responses of untested harmonic forcing scenarios, is straightforward with the proposed method, by applying linear modal analysis methods available in commercial software packages to measured constant-response FRFs of nonlinear structures. Finally, the proposed approach provides two different ways of determining near-resonant frequency response curves for untested constant-amplitude harmonic forcing scenarios; either computationally by using the nonlinear modal parameters identified during RCT, or experimentally by directly extracting isocurves of constant-amplitude forcing from the measured Harmonic Force Surface (HFS), a new concept proposed in this paper. It should be noted that both approaches are capable of determining unstable branches of frequency response curves, which may occur in strongly nonlinear systems. Theoretically, both approaches must give identical results, which constitutes a self-validation measure for the proposed method.

The paper is organized as follows. In Section 2, the theoretical background of the proposed approach is given. Subsequently, in Section 3 the proposed experimental methodology used to identify nonlinear modal parameters, and two different ways of determining near-resonant frequency responses corresponding to constant-amplitude harmonic forcing are explained in detail. Section 4 is dedicated to the numerical validation of the proposed approach with a lumped MDOF system with strong conservative nonlinearity. In Section 5, the method is applied on a real cantilever beam supported at its free end by thin metal strips which create cubic stiffness effect due to geometric nonlinearity, and also on a real missile structure which exhibits considerable nonlinear damping due to bolted joints. Finally, conclusions are discussed in Section 6.

\section{Theory}

\subsection{The nonlinearity matrix concept}

Equation of motion of a nonlinear $n$ degrees-of-freedom system with structural damping subjected to a harmonic excitation force of frequency $\omega$, neglecting all the sub- and super-harmonic terms, can be written in the form of a nonlinear complex algebraic equation in frequency domain as follows

$$
-\omega^{2}[M]\{X\}+i[H]\{X\}+[K]\{X\}+\left\{F_{N}\right\}=\{F\},
$$

where $[M],[H]$ and $[K]$ are the mass, hysteretic (structural) damping and stiffness matrices of the underlying linear system, respectively. All matrices are symmetric and positive definite, whereas $[K]$ and $[H]$ can be positive semi-definite as well. $\{X\}$, $\left\{F_{N}\right\}$ and $\{F\}$ are the complex vectors of displacement amplitude, nonlinear internal force amplitude and external excitation force amplitude, respectively.

According to Describing Function Method (DFM) [21], the complex vector of nonlinear internal force amplitude can be expressed as

$$
\left\{F_{N}\right\}=[\Delta]\{X\},
$$

where $[\Delta]$ is the displacement level dependent complex and symmetric nonlinearity matrix. Real and imaginary parts of this matrix correspond to the displacement level dependent nonlinear stiffness and nonlinear damping matrices, respectively.

Substituting Eq. (2) into Eq. (1) yields

$$
\left(-\omega^{2}[M]+i[H]+[K]+[\Delta]\right)\{X\}=\{F\} .
$$


It should be noted that DFM is mathematically equivalent to the classical Harmonic Balance Method (HBM). The major difference between DFM and HBM relies in the expression of nonlinear internal forces. HBM expresses nonlinear internal forces as a single force vector, whereas in DFM they are written as a multiplication of the so-called Nonlinearity Matrix with displacement amplitude vector as shown in Eq. (2). The second representation allows one to treat a nonlinear system, mathematically, as a linear system at a given displacement amplitude level, which makes it possible to extend some of the methods developed for linear systems to nonlinear systems by using iterative solutions.

\subsection{The nonlinear eigenvalue problem}

According to the single nonlinear mode theory [5-7], NNMs are found by solving the nonlinear eigenvalue problem associated with the conservative part of Eq. (3) as follows

$$
\left([K]+[\Delta]_{r e}\right)\left\{\bar{\psi}\left(q_{r}\right)\right\}_{r}=\bar{\omega}_{r}^{2}\left(q_{r}\right)[M]\left\{\bar{\psi}\left(q_{r}\right)\right\}_{r} .
$$

Here, $q_{r}$ is the $r$ th modal amplitude, $\left\{\bar{\psi}\left(q_{r}\right)\right\}_{r}$ and $\bar{\omega}_{r}\left(q_{r}\right)$ are the $r$ th real valued NNM and its corresponding natural frequency, which are functions of $q_{r}$. $[\Delta]_{r e}$ represents the real part of the nonlinearity matrix, which corresponds to the nonlinear stiffness matrix. It should be noted that $[\Delta]_{r e}$ depends on the displacement response level of the system, and therefore on the product $q_{r}\left\{\bar{\psi}\left(q_{r}\right)\right\}_{r}$, i.e.

$$
[\Delta]_{r e}=\left[\Delta\left(q_{r}\left\{\bar{\psi}\left(q_{r}\right)\right\}_{r}\right)\right]_{r e} .
$$

Consequently, Eq. (4) is a nonlinear eigenvalue problem which requires an iterative solution procedure. Starting from the corresponding normal mode of the underlying linear system, Eq. (4) can be solved for $\left\{\bar{\psi}\left(q_{r}\right)\right\}_{r}$ and $\bar{\omega}_{r}\left(q_{r}\right)$ by using various iterative solution techniques (e.g. Newton-Raphson Method [6]).

\subsection{The single nonlinear mode method}

If the modes are well separated and no pronounced modal coupling occurs in the energy range of interest, near-resonant solution of Eq. (3) around an $r$ th mode can be approximated by a single NNM calculated from Eq. (4) as follows [5-7]

$$
\{X\}=q_{r}\left\{\bar{\psi}\left(q_{r}\right)\right\}_{r} .
$$

Substituting Eq. (6) into Eq. (3) and premultiplying by $\left\{\bar{\psi}\left(q_{r}\right)\right\}_{r}^{T}$ yields

$$
\left(-\omega^{2} \bar{m}_{r}\left(q_{r}\right)+\bar{k}_{r}\left(q_{r}\right)+i \bar{h}_{r}\left(q_{r}\right)\right) q_{r}=\left\{\bar{\psi}\left(q_{r}\right)\right\}_{r}^{T}\{F\}
$$

where

$$
\begin{aligned}
& \bar{m}_{r}\left(q_{r}\right)=\left\{\bar{\psi}\left(q_{r}\right)\right\}_{r}^{T}[M]\left\{\bar{\psi}\left(q_{r}\right)\right\}_{r}, \\
& \bar{k}_{r}\left(q_{r}\right)=\left\{\bar{\psi}\left(q_{r}\right)\right\}_{r}^{T}\left([K]+[\Delta]_{r e}\right)\left\{\bar{\psi}\left(q_{r}\right)\right\}_{r}, \\
& \bar{h}_{r}\left(q_{r}\right)=\left\{\bar{\psi}\left(q_{r}\right)\right\}_{r}^{T}\left([H]+[\Delta]_{i m}\right)\left\{\bar{\psi}\left(q_{r}\right)\right\}_{r} .
\end{aligned}
$$

Here, $\bar{m}_{r}\left(q_{r}\right), \bar{k}_{r}\left(q_{r}\right)$ and $\bar{h}_{r}\left(q_{r}\right)$ are the modal mass, modal stiffness and modal hysteretic damping, respectively. $[\Delta]_{i m}$ represents the imaginary part of the nonlinearity matrix, which corresponds to the displacement response level dependent nonlinear damping matrix.

Eq. (7) can be alternatively written as

$$
\bar{m}_{r}\left(q_{r}\right)\left(-\omega^{2}+\bar{\omega}_{r}^{2}\left(q_{r}\right)+i \bar{\eta}_{r}\left(q_{r}\right) \bar{\omega}_{r}^{2}\left(q_{r}\right)\right) q_{r}=\left\{\bar{\psi}\left(q_{r}\right)\right\}_{r}^{T}\{F\}
$$

where

$$
\bar{\omega}_{r}^{2}\left(q_{r}\right)=\frac{\bar{k}_{r}\left(q_{r}\right)}{\bar{m}_{r}\left(q_{r}\right)}, \quad \bar{\eta}_{r}\left(q_{r}\right)=\frac{\bar{h}_{r}\left(q_{r}\right)}{\bar{m}_{r}\left(q_{r}\right) \bar{\omega}_{r}^{2}\left(q_{r}\right)} .
$$

Modal amplitude $q_{r}$ can be solved from Eq. (9) as follows

$$
q_{r}=\frac{\left\{\bar{\psi}\left(q_{r}\right)\right\}_{r}^{T}\{F\}}{\bar{m}_{r}\left(q_{r}\right)\left(\bar{\omega}_{r}^{2}\left(q_{r}\right)-\omega^{2}+i \bar{\eta}_{r}\left(q_{r}\right) \bar{\omega}_{r}^{2}\left(q_{r}\right)\right)} .
$$

Inserting Eq. (11) into Eq. (6) yields 


$$
\{X\}=\frac{\left\{\bar{\psi}\left(q_{r}\right)\right\}_{r}\left\{\bar{\psi}\left(q_{r}\right)\right\}_{r}^{T}\{F\}}{\bar{m}_{r}\left(q_{r}\right)\left(\bar{\omega}_{r}^{2}\left(q_{r}\right)-\omega^{2}+i \bar{\eta}_{r}\left(q_{r}\right) \bar{\omega}_{r}^{2}\left(q_{r}\right)\right)} .
$$

Here, NNM can be normalized with respect to modal mass as follows

$$
\left\{\bar{\phi}\left(q_{r}\right)\right\}_{r}=\frac{\left\{\bar{\psi}\left(q_{r}\right)\right\}_{r}}{\sqrt{\bar{m}_{r}\left(q_{r}\right)}} .
$$

Substituting Eq. (13) into Eq. (12) yields

$$
\{X\}=\frac{\left\{\bar{\phi}\left(q_{r}\right)\right\}_{r}\left\{\bar{\phi}\left(q_{r}\right)\right\}_{r}^{T}\{F\}}{\bar{\omega}_{r}^{2}\left(q_{r}\right)-\omega^{2}+i \bar{\eta}_{r}\left(q_{r}\right) \bar{\omega}_{r}^{2}\left(q_{r}\right)} .
$$

Near-resonant receptance $\bar{\alpha}_{j k}$ at point $j$ for a given excitation at point $k$ can be deduced from Eq. (14) as follows

$$
\bar{\alpha}_{j k}\left(\omega, q_{r}\right)=\frac{\bar{\phi}_{j r}\left(q_{r}\right) \bar{\phi}_{k r}\left(q_{r}\right)}{\bar{\omega}_{r}^{2}\left(q_{r}\right)-\omega^{2}+i \bar{\eta}_{r}\left(q_{r}\right) \bar{\omega}_{r}^{2}\left(q_{r}\right)} .
$$

In Eq. (15) the nonlinear hysteretic modal damping model can be replaced by an equivalent nonlinear viscous damping model as well. Accordingly, an alternative form of Eq. (15) can be written as follows

$$
\bar{\alpha}_{j k}\left(\omega, q_{r}\right)=\frac{\bar{\phi}_{j r}\left(q_{r}\right) \bar{\phi}_{k r}\left(q_{r}\right)}{\bar{\omega}_{r}^{2}\left(q_{r}\right)-\omega^{2}+i 2 \bar{\xi}_{r}\left(q_{r}\right) \omega \bar{\omega}_{r}\left(q_{r}\right)},
$$

where $\bar{\xi}_{r}\left(q_{r}\right)$ is the nonlinear viscous modal damping ratio.

The experimental methodology proposed to identify nonlinear modal parameters which can be used for the synthesis of near-resonant frequency response curves for untested constant-amplitude harmonic forcing scenarios is explained in the next section.

\section{Proposed experimental methodology - Identification of nonlinear modal parameters and synthesis of frequency responses for constant-amplitude harmonic forcing}

\subsection{Measurement of constant-response FRFs in quasi-linear form}

Modal parameters in Eqs. (15) and (16) are functions of a single parameter; the modal amplitude. In this paper, it is proposed to measure constant-response FRFs of nonlinear systems by keeping the driving point displacement amplitude constant with RCT strategy. According to the single nonlinear mode assumption, these constant-response FRFs are expected to come out in quasi-linear form. Consequently, modal parameters can be extracted from measured constant-response FRFs by using standard linear modal analysis methods available in commercial software packages. This approach essentially extends the method proposed by Arslan and Özgüven [22] which is restricted to structures where nonlinearity is localized between a single DOF and ground, to complex engineering structures with multiple nonlinearities connecting internal coordinates and spread over the structure (e.g., structures with several bolted joints).

Although single nonlinear mode theory [5] completely discards higher harmonics in the derivation of the nonlinear receptance model given in Eqs. (15) and (16), quasi-linearization of FRFs with RCT strategy can still be achieved under the effects of higher harmonics if these effects are not pronounced. In Section 4, this is demonstrated on the RCT simulation of a lumped MDOF system with strong conservative nonlinearity, where the system is excited at a single DOF and the fundamental harmonic displacement of the driving point is kept constant. Firstly, the frequency responses of the fundamental and higher harmonics as well as excitation force spectrum are calculated by using multi-harmonics version of the DFM [23]. Secondly, constant-response FRFs corresponding to the fundamental harmonic are determined by dividing the fundamental harmonic displacement with the harmonic excitation force. Eventually, it is shown that FRFs determined by using multiple harmonics are also quasi-linear.

\subsection{Excitation strategy}

Several experimental studies have shown that in the absence of internal resonances; single point, single harmonic excitation is sufficient to isolate nonlinear modes to a satisfying accuracy [14,19]. Furthermore, in case of a single point excitation, keeping the modal amplitude constant is equivalent to keeping the displacement amplitude of the driving point constant. Due to these practical benefits, in the proposed method, a single input stepped-sine test strategy is used to identify modal parameters. Theoretically, RCT approach can also be applied by using multi-point excitation. In that case, adjustment of the amplitude ratios of excitation signals would be necessary, as in the case of NNM force appropriation, to keep the modal amplitude at a constant level. This adjustment can be achieved with the help of a preliminary single point excitation test. 


\subsection{Response control strategy}

Displacement amplitude of the driving point can be kept constant either directly or indirectly depending on the type of the sensor used during RCT. Since accelerometer is the most widely used sensor in experimental modal analysis, it was chosen as the control sensor in the proposed method. Acceleration profile corresponding to a constant displacement amplitude over the frequency range of interest is calculated and input to the closed-loop controller as a reference profile, which is an available option in standard modal testing software packages (e.g. LMS Test Lab®).

\subsection{Identification of nonlinear modal parameters and experimental extraction of mass normalized NNMs}

Determination of near-resonant frequency response curves for various unmeasured harmonic forcing scenarios is an important task to understand complex dynamics of engineering structures and eventually to satisfy critical design requirements. If one could experimentally extract nonlinear modal parameters used in Eqs. (15) and (16), these identified modal parameters can then be used to synthesize near-resonant frequency responses corresponding to various constantamplitude harmonic forcing scenarios.

In the proposed method, in order to identify nonlinear modal parameters, constant-response FRFs at several different displacement amplitude levels are measured by conducting a series of modal tests with RCT strategy. Then, modal identification is achieved by fitting the following analytical model to the measured constant-response FRFs with an appropriate linear modal analysis method

$$
\bar{\alpha}_{j k}\left(\omega, q_{r}\right)=\frac{\bar{A}_{j k r}\left(q_{r}\right)}{\bar{\omega}_{r}^{2}\left(q_{r}\right)-\omega^{2}+i \bar{\eta}_{r}\left(q_{r}\right) \bar{\omega}_{r}^{2}\left(q_{r}\right)},
$$

where $A_{j k r}\left(q_{r}\right)$ is a complex valued modal constant. The nonlinear hysteretic modal damping model used in Eq. (17) can be replaced by an equivalent nonlinear viscous modal damping model as follows

$$
\bar{\alpha}_{j k}\left(\omega, q_{r}\right)=\frac{\bar{A}_{j k r}\left(q_{r}\right)}{\bar{\omega}_{r}^{2}\left(q_{r}\right)-\omega^{2}+i 2 \bar{\xi}_{r}\left(q_{r}\right) \omega \bar{\omega}_{r}\left(q_{r}\right)} .
$$

Once $A_{j k r}\left(q_{r}\right), \bar{\omega}_{r}\left(q_{r}\right)$ and $\bar{\xi}_{r}\left(q_{r}\right)$ (or $\bar{\eta}_{r}\left(q_{r}\right)$ ) are experimentally extracted, they can be plotted with respect to modal amplitude. These plots can either be used in parametric form by fitting polynomials, if possible, or directly as look-up tables in the synthesis of near-resonant frequency response curves for untested harmonic forcing scenarios.

In case of a single input and multiple outputs modal testing, mass normalized NNMs can be determined from experiment by using the following procedure.

First of all, identified modal constants can be collected into a vector as follows

$$
\left\{\bar{A}\left(q_{r}\right)\right\}_{r}^{T}=\left\{\begin{array}{llll}
\bar{A}_{11 r}\left(q_{r}\right) & \bar{A}_{21 r}\left(q_{r}\right) & \ldots & \bar{A}_{m 1 r}\left(q_{r}\right)
\end{array}\right\}^{T}
$$

where $m$ indicates the total number of measurement points.

Comparison of Eqs. (17) and (18) with Eqs. (15) and (16) reveals that experimentally extracted modal constants are closely related to mass normalized NNMs with the following relation

$$
\bar{A}_{j k r}\left(q_{r}\right)=\bar{\phi}_{j r}\left(q_{r}\right) \bar{\phi}_{k r}\left(q_{r}\right) \text {. }
$$

Substituting Eq. (20) into Eq. (19) yields

$$
\left\{\bar{A}\left(q_{r}\right)\right\}_{r}=\left\{\bar{\phi}_{1 r}\left(q_{r}\right) \bar{\phi}_{1 r}\left(q_{r}\right) \quad \bar{\phi}_{1 r}\left(q_{r}\right) \bar{\phi}_{2 r}\left(q_{r}\right) \quad \ldots \quad \bar{\phi}_{1 r}\left(q_{r}\right) \bar{\phi}_{m r}\left(q_{r}\right)\right\}^{T} .
$$

It can easily be noticed that all terms of the vector given in Eq. (21) have a common multiplier which is $\bar{\phi}_{1 r}\left(q_{r}\right)$. This multiplier can be determined by taking square root of the identified modal constant of the driving point FRF as follows

$$
\bar{\phi}_{1 r}\left(q_{r}\right)=\sqrt{\bar{A}_{11 r}\left(q_{r}\right)} \text {. }
$$

Finally, dividing Eq. (21) by the square root term given in Eq. (22), the NNM of interest can be identified in mass normalized form as follows

$$
\left\{\bar{\phi}\left(q_{r}\right)\right\}_{r}=\frac{1}{\sqrt{\bar{A}_{11 r}\left(q_{r}\right)}}\left\{\bar{A}\left(q_{r}\right)\right\}_{r} .
$$

The nonlinear modal damping ratio and mass normalized NNMs are essential elements required in the synthesis of frequency response curves for untested harmonic forcing scenarios. Obviously, if one is interested in the synthesis of the frequency response curves of a single point, identification of the modal constant corresponding to that point will be sufficient.

In concluding this section, it is important to note that the NNMs used in Eqs. (15) and (16) are assumed to be the NNMs of the underlying conservative system, whereas those extracted from experimental data by using Eq. (23) are the NNMs of the 
actual damped system, which may seem paradoxical. However, the damped NNMs of a nonlinear system can be approximated by the NNMs of the underlying undamped system, and vice versa, under the assumption of moderate damping as discussed in [15,24], and as also experimentally confirmed in this study. The applicability of the method for high damping will be the subject of a future work.

\subsection{Synthesis of frequency responses for untested constant-amplitude harmonic forcing scenarios}

The nonlinear modal parameters identified from constant-response FRFs can be employed in the following equation to calculate near-resonant frequency responses for untested constant-amplitude harmonic forcing scenarios

$$
X_{j}\left(q_{r}\right)=\frac{\bar{A}_{j k r}\left(q_{r}\right) F_{k}}{\bar{\omega}_{r}^{2}\left(q_{r}\right)-\omega^{2}+i 2 \bar{\xi}_{r}\left(q_{r}\right) \omega \bar{\omega}_{r}\left(q_{r}\right)},
$$

where $X_{j}\left(q_{r}\right)$ is the displacement amplitude at point $j$ for a given constant-amplitude $F_{k}$ at point $k$.

In this study, Eq. (24) is solved iteratively by using Newton's Method with arc-length continuation algorithm which is capable of capturing any unstable branch which might occur in strongly nonlinear systems. Eq. (24) can also be solved explicitly in closed form for the excitation frequency as shown in [25].

\subsection{Experimental extraction of frequency responses for untested constant-amplitude harmonic forcing scenarios by using HFS}

As an alternative to the computational approach given in Eq. (24), the HFS concept is capable of deriving frequency response curves, experimentally, at constant force levels including unstable branches, if there is any, by using harmonic force spectra measured at constant vibration levels during RCT without any extra experimental effort.

\subsubsection{Difficulties in frequency response measurement of nonlinear structures with standard force-control}

In linear experimental modal analysis, standard closed-loop stepped sine test with force control starts with a linear system identification which determines a rough initial estimate of the plant, i.e. the transfer function of the structure under test. In case of nonlinear structures, this procedure may lead to very poor estimate of the structure's actual dynamic behavior. Consequently, controller may need to make many corrective iterations to keep the force within tolerance of the reference profile, which may become very time consuming. Furthermore, in case of nonlinear structures with strong stiffening or softening character, multiple steady-state vibration responses may co-exist for the same excitation frequency, which results in an overhanging unstable branch in the frequency response. Standard force-control algorithms available in commercial softwares can only measure nonlinear frequency responses until the turning points of the stable branches, at the cost of multiple runs with different sweep directions. When a turning point is reached, standard force-controller cannot avoid jumping from one stable branch to another, passing over the unstable region. Even worse, in some cases small corrective force perturbations of the controller to capture the reference signal in the vicinity of the turning points may lead to a premature jump before reaching the actual turning point. These drawbacks of standard techniques paved the way for the development of control algorithms such as PLL and CBC. The primary advantage of these control strategies over classical force-control approach is the ability of measuring backbone curves and unstable branches of nonlinear frequency responses. However, these methods were not commercialized yet, therefore available standard equipment cannot be used in these approaches. As an alternative to new generation control strategies, the Harmonic Force Surface (HFS) concept proposed in this paper can be used as described below, in order to determine unstable branches of nonlinear frequency response curves by using standard controllers available in commercial modal testing hardware and driven by commercial software together with RCT strategy.

\subsubsection{HFS approach}

In case of strong stiffness nonlinearity where multiple steady-state vibration responses co-exist for the same excitation frequency, response level of the structure highly depends on initial conditions. In case of force-control test strategy, there is no control over vibration response which may exhibit drastic changes around resonance even if very small frequency steps are used. Since gradual change in vibration response and eventually in initial conditions are not guaranteed, experimental continuation of the unstable branch cannot be achieved. In RCT, this problem is solved by keeping the displacement amplitude constant, which results in smooth response spectrum incorporating points on the unstable branch as well. This is somewhat different from PLL and CBC algorithms which aim tracing points on the unstable branch consecutively. In RCT, points on the unstable branch are visited at different times during stepped-sine tests carried out at different vibration amplitude levels. Then they are collected together on the HFS. Determination of the nonlinear frequency response with unstable branch by using RCT and HFS consists of the following steps:

1. An RCT is carried out by keeping the displacement amplitude of the driving point constant. The test is repeated at several different displacement amplitude levels (in general, repeating the test at 10-15 different levels may be sufficient to obtain satisfactory results), and the corresponding harmonic force spectra of the driving point are measured.

2. Harmonic Force Surface, HFS, is constructed by collecting measured harmonic force spectra and using linear interpolation. 
3. The contour plot obtained by cutting the HFS with a constant force plane gives the harmonic response spectrum corresponding to that force level, including any unstable branch if there is any.

Application of the HFS procedure given above is demonstrated with numerical and experimental case studies in the subsequent sections.

\section{Numerical validation}

In this section, the proposed modal identification method is validated on the 5 DOF nonlinear lumped system with 5 cubic stiffness elements, which is shown in Fig. 1 . Systems parameters are as follows: $m=1 \mathrm{~kg}, k=10000 \mathrm{~N} / \mathrm{m}, c=5 \mathrm{Ns} / \mathrm{m}$, $k^{*}=10^{7} \mathrm{~N} / \mathrm{m}^{3}$.

Standard force-control and RCT simulations carried out in this section are achieved by solving the following equation of motion which includes multiple harmonics [23]

$$
\left(\left[\begin{array}{cccc}
{[Z]_{11}} & 0 & \cdots & 0 \\
0 & {[Z]_{22}} & \cdots & 0 \\
\vdots & \vdots & \vdots & \vdots \\
0 & 0 & \cdots & {[Z]_{h h}}
\end{array}\right]+\left[\begin{array}{cccc}
{[\Delta]_{11}} & {[\Delta]_{12}} & \cdots & {[\Delta]_{1 h}} \\
{[\Delta]_{21}} & {[\Delta]_{22}} & \cdots & {[\Delta]_{2 h}} \\
\vdots & \vdots & \vdots & \vdots \\
{[\Delta]_{h 1}} & {[\Delta]_{h 2}} & \cdots & {[\Delta]_{h h}}
\end{array}\right]\right)\left\{\begin{array}{c}
\{X\}_{1} \\
\{X\}_{2} \\
\vdots \\
\{X\}_{h}
\end{array}\right\}=\left\{\begin{array}{c}
\{F\} \\
0 \\
\vdots \\
0
\end{array}\right\}
$$

where $\{X\}_{h}$ and $[Z]_{h h}$ are the complex displacement amplitude vector and the dynamic stiffness matrix corresponding to the $h$ th harmonic, respectively. $\{F\}$ denotes the vector of external excitation force amplitude. $[\Delta]_{11},[\Delta]_{12}$ etc. are the components of the complex nonlinearity matrix, where off-diagonal matrices represent coupling terms between different harmonics (e.g., $[\Delta]_{12}$ is the coupling matrix between 1st and 2nd harmonics). In the paper where DFM was first introduced [21], only a single harmonic was considered, which corresponds to taking $[Z]_{11}$ and $[\Delta]_{11}$ matrices into account and neglecting all other submatrices in Eq. (25). $[Z]_{h h}$ can be explicitly written as follows

$$
[Z]_{h h}=-(h \omega)^{2}[M]+i[H]+[K] .
$$

In the force-control simulations, a single point, single harmonic excitation force is applied to 1st DOF which is the driving point, and Eq. (25) is solved for the displacement amplitudes of the fundamental and higher harmonics by keeping the amplitude of the excitation force constant. On the other hand, in the RCT simulations, the displacement amplitude corresponding to the first harmonic of the 1st DOF is kept constant and Eq. (25) is solved for the displacement amplitudes of all other DOFs and higher harmonics as well as the amplitude of the excitation force. In both simulations, equation of motion is solved numerically by using Newton's Method with arc-length continuation algorithm. In this case study, it is found to be sufficient to consider only the first and the third harmonics in the solution. The effect of the third harmonic on the FRFs in the first mode is illustrated in Fig. 2. It should be noted that throughout the paper, FRF term is used to refer to the frequency response function between the fundamental harmonic response and the excitation forcing. Since FRFs are not invariant of the excitation forcing in nonlinear systems, normalization of the frequency response with the amplitude of the excitation forcing may seem to be unnecessary. However, normalization process, which is very commonly used even for nonlinear systems, in literature, helps to suppress the effect of excitation forcing on the amplitude of the frequency response curve and to emphasize the effect of nonlinear stiffness and damping on the shape change of the frequency response curve.

\subsection{Results of the standard force-control simulation}

The classical approach to measure frequency responses of a nonlinear system is to apply force-controlled stepped-sine testing by keeping the amplitude of the harmonic forcing constant throughout the frequency sweep. Accordingly, a series of force-control simulations was carried out on the 5 DOF nonlinear system shown in Fig. 1. Constant-force FRFs determined around the first mode of the system at different forcing levels ranging from $1 \mathrm{~N}$ to $50 \mathrm{~N}$ are shown in Fig. 3. Obviously, the strong stiffness nonlinearity of the system changes the shape of each FRF considerably with increasing forcing level and

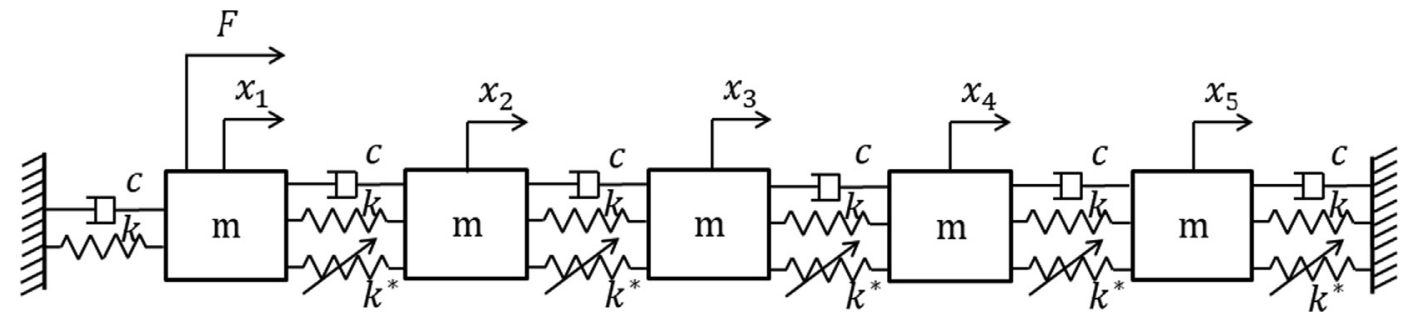

Fig. 1. 5 DOF system with cubic stiffness nonlinearity. 


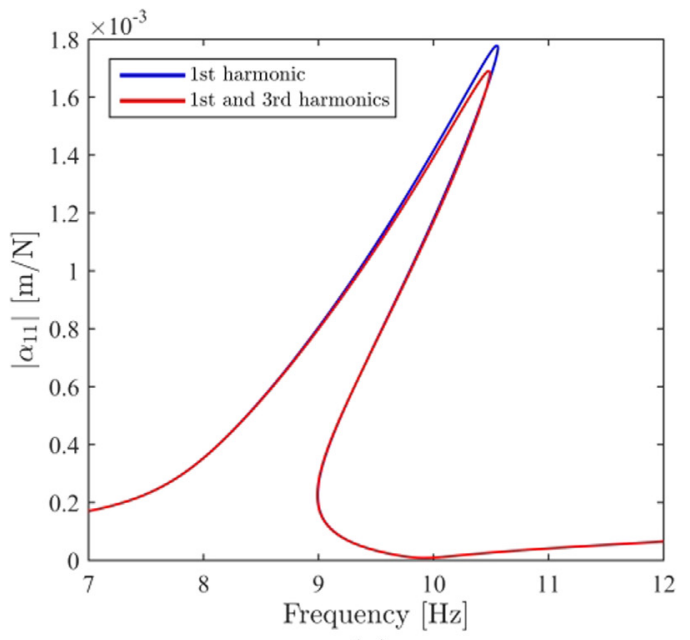

(a)

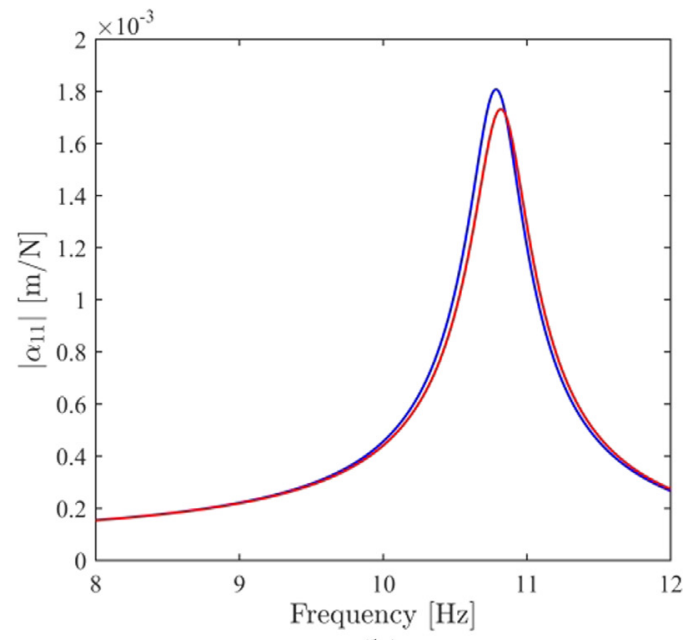

(b)

Fig. 2. Effect of the 3rd harmonic on the FRFs (a) constant-force driving point FRF at $50 \mathrm{~N}$ (b) constant-response driving point FRF at $0.10 \mathrm{~m}$.
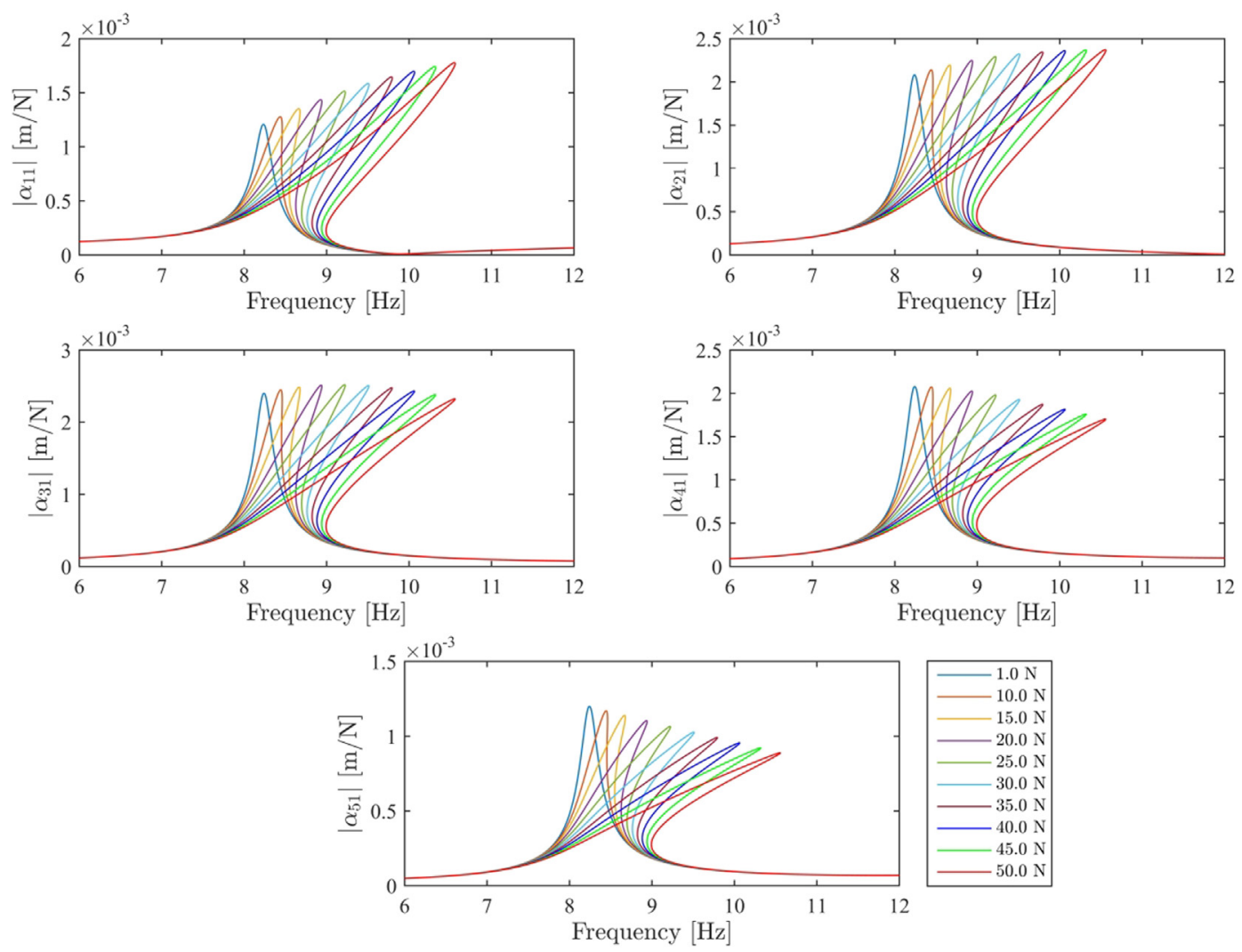

Fig. 3. Constant-force FRFs of the 5 DOF system with cubic stiffness, obtained by force-controlled stepped-sine testing simulations.

results in overhanging unstable branches. By virtue of the arc-length continuation algorithm, the computer simulation made it possible to capture these unstable branches and consequently, to determine exact locations of resonance points which constitute the NNM backbone curves. However, in real experimental applications, this cannot be achieved by using standard force control testing due to the jump phenomenon as explained in Section 3.6.1. In the following sections, it is shown that by just switching the control strategy from force control to response control, jump phenomenon can be avoided and consequently, standard equipment can still be used for modal identification of nonlinear systems. 


\subsection{Determination of constant-response FRFs by using the RCT simulation}

Harmonic excitation force spectra of the driving point (1st DOF) determined from RCT simulations at different displacement amplitude levels are shown in Fig. 4. By dividing the displacement amplitude spectrum of each DOF with the harmonic excitation force spectrum, constant-response FRFs (corresponding to the first harmonic) were determined around the first mode of the system at different displacement amplitude levels ranging from $0.01 \mathrm{~m}$ to $0.1 \mathrm{~m}$ as shown in Fig. 5 . It should be noted that not only constant-response FRFs of the 1st DOF, but also the constant-response FRFs of all other DOFs turned out to be quasi-linear. Of course, visual inspection is not sufficient, and a solid proof is given in the next section by fitting linear analytical model to constant-response FRFs. This outcome validates the fundamental hypothesis of the proposed method, i.e. if the modal amplitude is kept constant, FRFs given in Eqs. (15) and (16) turn out to be quasi-linear. It should be noted that in case of single point excitation, modal amplitude can be kept constant by just keeping the displacement amplitude of the driving point constant.

The physics behind the quasi-linearization phenomenon is essentially very simple. When the displacement amplitude of the driving point is kept constant, if the NNM of interest is well separated from the other modes and no internal resonances occur, then the near-resonant deflection shape of the system can be approximated by the product of that single NNM with the modal amplitude. If the modal amplitude is kept constant, the deflection shape and so the energy level of the system is frozen throughout the frequency sweep in the vicinity of the resonant region. Accordingly, if this frozen deflection shape is fed into the nonlinearity matrix given in Eq. (3), this matrix remains also constant, which quasi-linearizes the equation of motion. Even though the displacement level is kept constant through the frequency sweep, the resonant peaks are observed in the constant-response FRFs shown in Fig. 5. These peaks result from the dip of the excitation force spectrum at the resonant point as shown in Fig. 4.

It is important to note that quasi-linearization holds true even when the shape of the NNM strongly depends on the response level as shown in Fig. 6. The shape of the 1st NNM of the 5 DOF nonlinear system considered in this study deviates from a symmetric shape toward an asymmetric shape with increasing displacement amplitude level due to the missing cubic stiffness element between 1st DOF and ground as shown in Fig. 1. Yet, constant-response FRFs given in Fig. 5 are still quasilinear.

A final important observation made from Fig. 5 is that constant-response FRFs do not exhibit unstable branches although they cover the excitation forcing levels $(1 \mathrm{~N}$ to $50 \mathrm{~N}$ ) studied in Section 4.1. As explained in Section 3.6.2, the points on the unstable branches are visited at different times during RCT carried out at different displacement amplitude levels. In other words, points on the unstable branch of a constant-force FRF given in Fig. 3 are stabilized and shared among several different constant-response FRFs given in Fig. 5. This point is explained further in Section 4.5 by using the HFS concept.

\subsection{Identification of nonlinear modal parameters from quasi-linear constant-response FRFs}

Once constant-response FRFs are determined by using RCT simulations as shown in Fig. 5, modal identification methods for linear systems can then be used to extract nonlinear modal parameters as a function of modal amplitude. In this study, well known peak-picking method is used to determine modal parameters at each displacement amplitude level. As an example, constant-response FRFs corresponding to the highest displacement amplitude level, i.e. $0.10 \mathrm{~m}$, obtained by RCT simulation are compared with the corresponding FRFs synthesized by using modal parameters identified from peak-picking technique in Fig. 7. The good match between the results of the simulated experiment and the corresponding linear analytical models shows that constant-response FRFs are really quasi-linear.

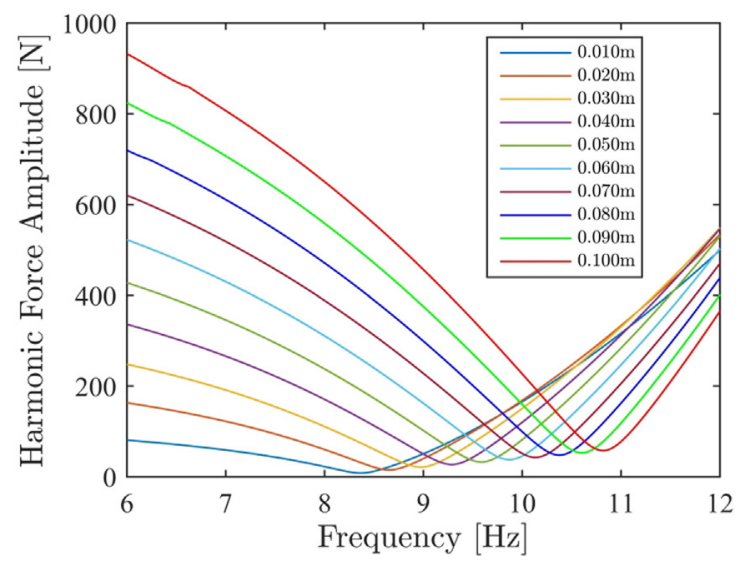

Fig. 4. Harmonic force spectra of the driving point (1st DOF) of the 5 DOF nonlinear system obtained from RCT simulations. 

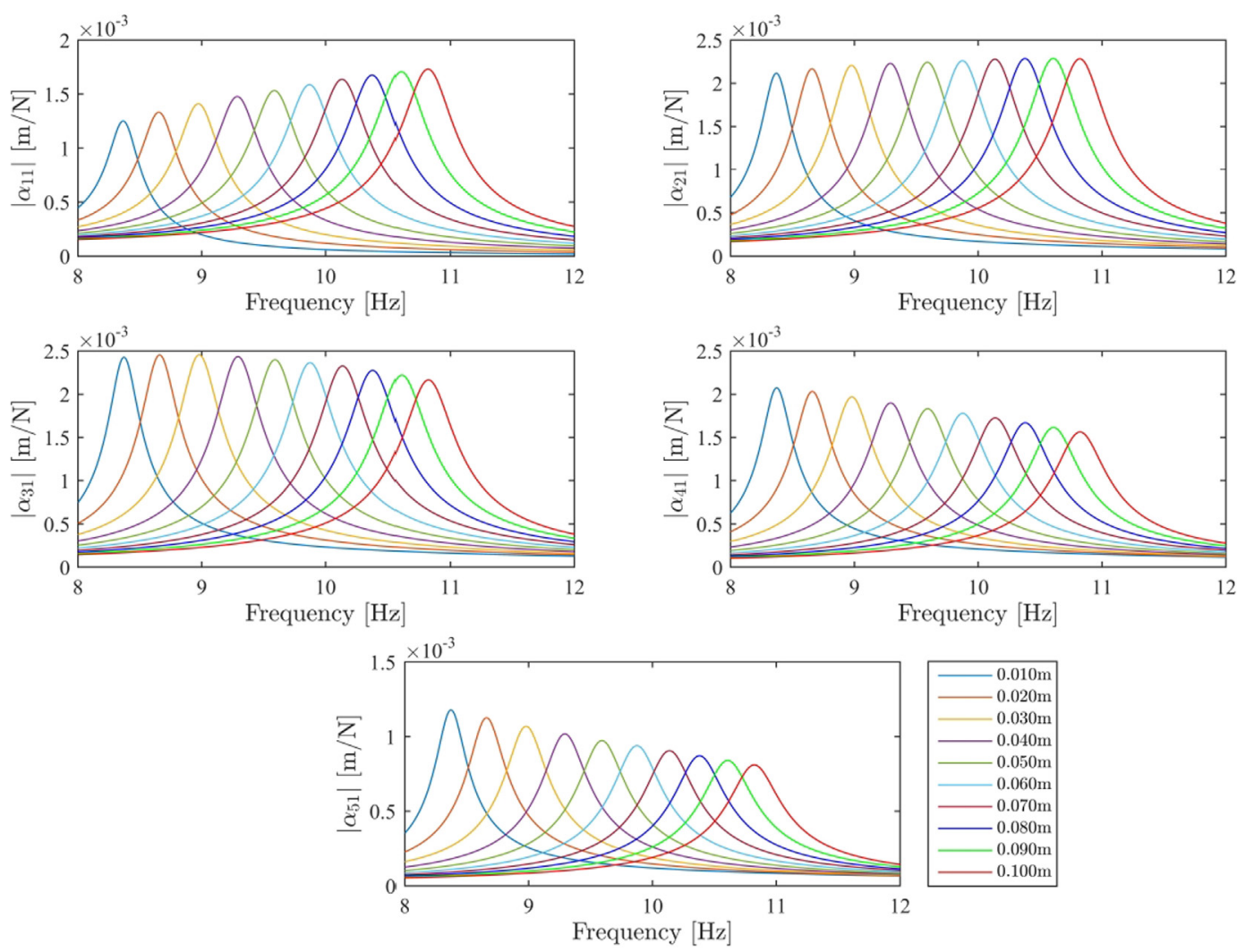

Fig. 5. Constant-response FRFs of the 5 DOF system with cubic stiffness, obtained from RCT simulations.

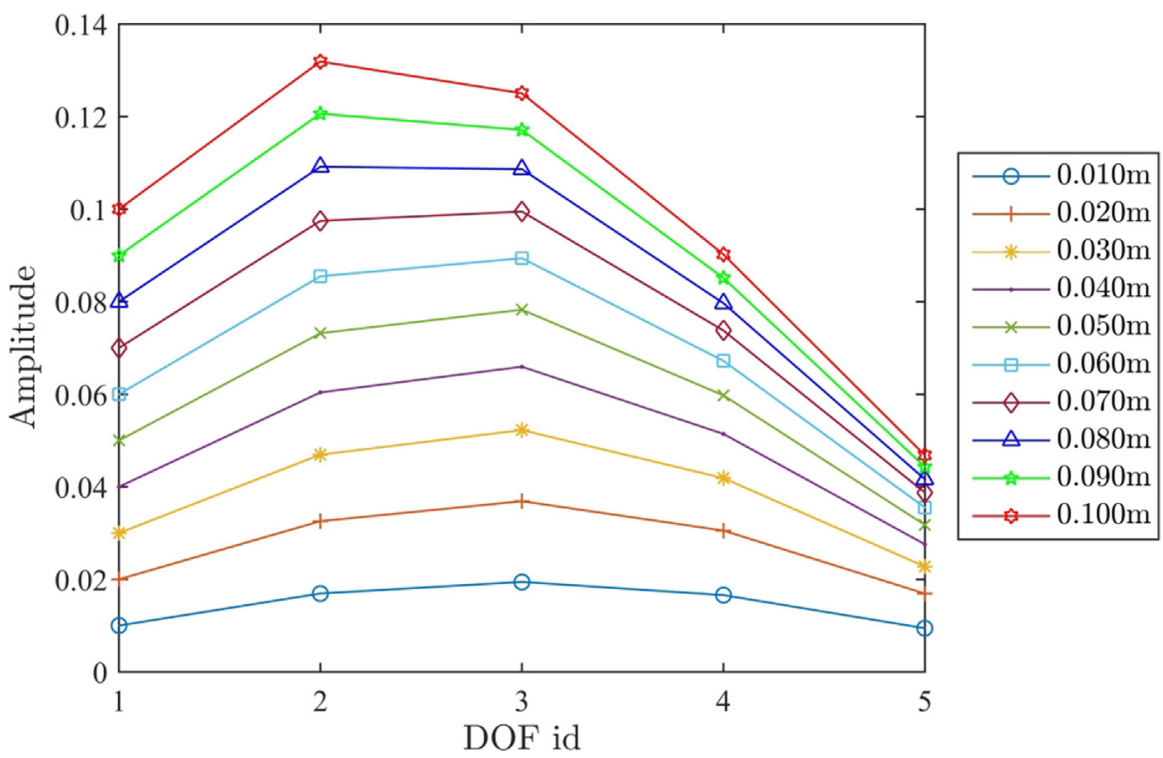

Fig. 6. The shape change of the 1 st NNM of the 5 DOF nonlinear system with increasing displacement amplitude level.

By processing each set of FRFs corresponding to each displacement amplitude level (see Fig. 5) and collecting modal parameters of the 1st mode; the variations of the natural frequency, the nonlinear modal damping ratio and the modal constants with respect to modal amplitude can be determined as shown in Fig. 8 and Fig. 9. Substituting these modal parameters into Eq. (24), one can predict frequency responses of the system to various forcing scenarios without making any force con- 

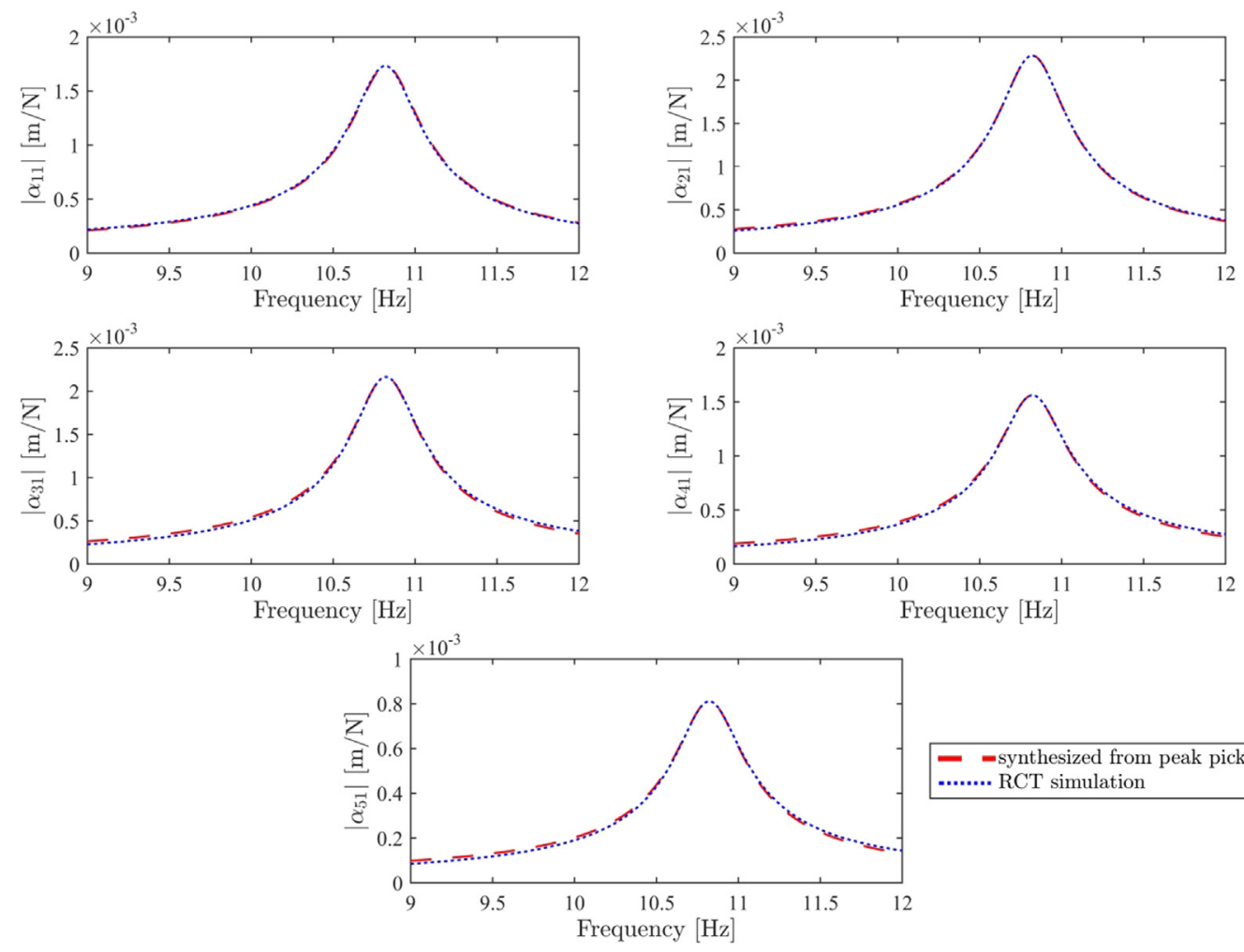
- $=$ synthesized from peak picking method
........ RCT simulation

Fig. 7. Comparison of the constant-response FRFs obtained from RCT simulation, with the FRFs synthesized by using modal parameters identified from pick-peaking method at $0.10 \mathrm{~mm}$ amplitude level.

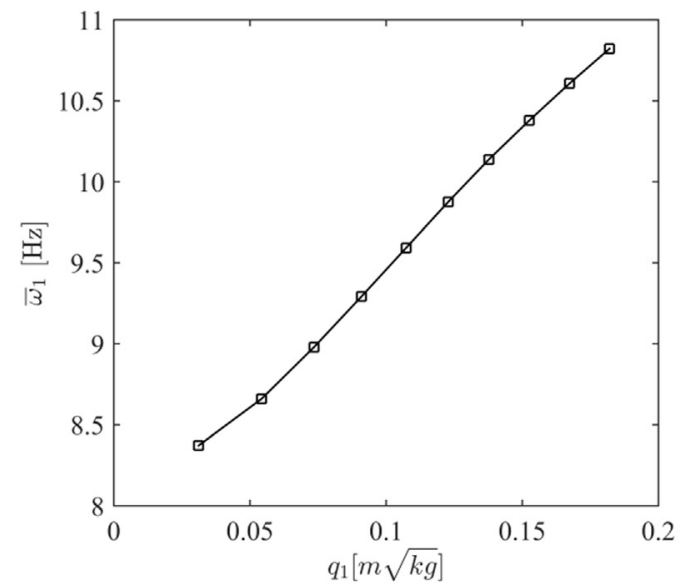

(a)

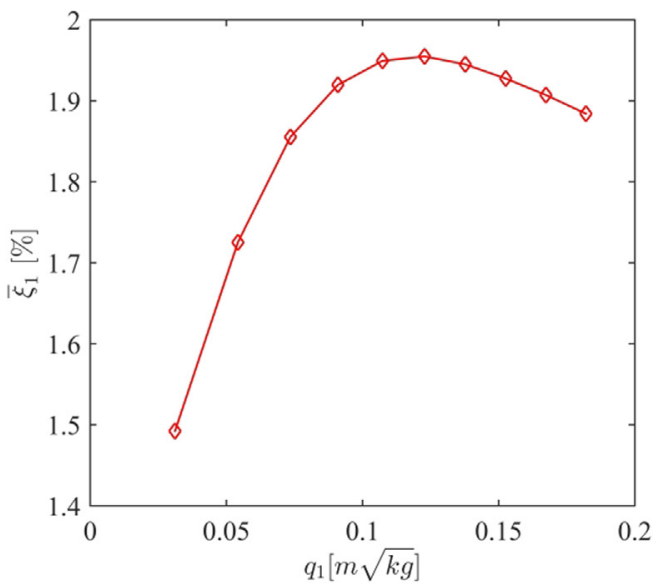

(b)

Fig. 8. Variation of the modal parameters corresponding to the 1 st mode of the 5 DOF nonlinear system with modal response level (a) natural frequency (b) viscous modal damping ratio.

trolled test, which is very useful for the design and analysis of engineering structures. It should be noted that modal constants $A_{j k r}$ given in Fig. 9 are closely related with mass normalized NNMs as shown in Eq. (23) and explained in Section 3.4. So, RCT approach makes it possible to experimentally extract mass normalized NNM from the identified modal constants given in Fig. 9.

\subsection{Synthesis of constant-force FRFs by using the nonlinear modal parameters identified from RCT}

Identified modal parameters given in Fig. 8 and Fig. 9 can be employed in Eq. (24) to synthesize frequency responses for various harmonic forcing scenarios. For validation, frequency responses of all 5 dofs were synthesized at $50 \mathrm{~N}$ using Eq. (24) 


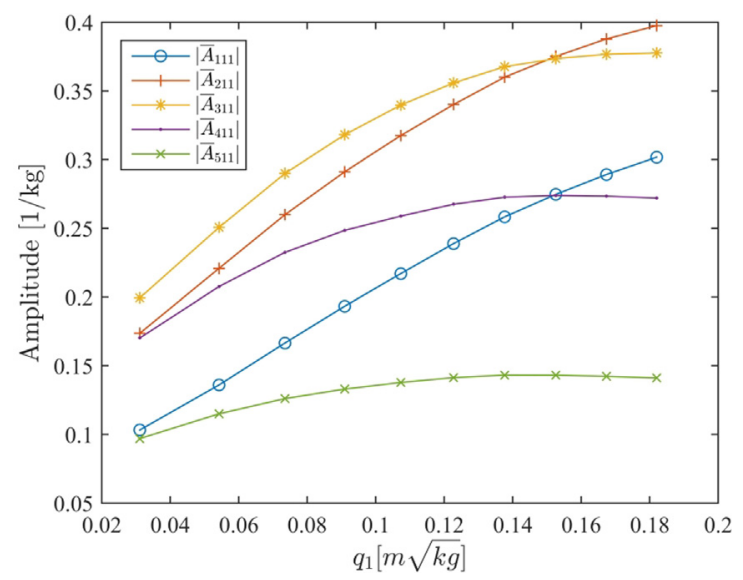

Fig. 9. Variation of the modal constants corresponding to the 1 st mode of the 5 DOF nonlinear system with modal response level.

iteratively by applying Newton's Method with arc-length continuation algorithm. These frequency responses were then normalized with $50 \mathrm{~N}$ and were compared with the results of constant-force simulation as shown in Fig. 10. The comparison was also made at different force levels as shown in Fig. 11. The match between the computational results and the simulated experimental results is found to be satisfactory. The deviation further away from resonance peaks results from discarding the contribution of higher modes, which is the basic assumption of the single nonlinear mode theory. It is important to note that the modal parameters given in Fig. 8 and Fig. 9 were used as look-up tables without necessitating any polynomial fit.
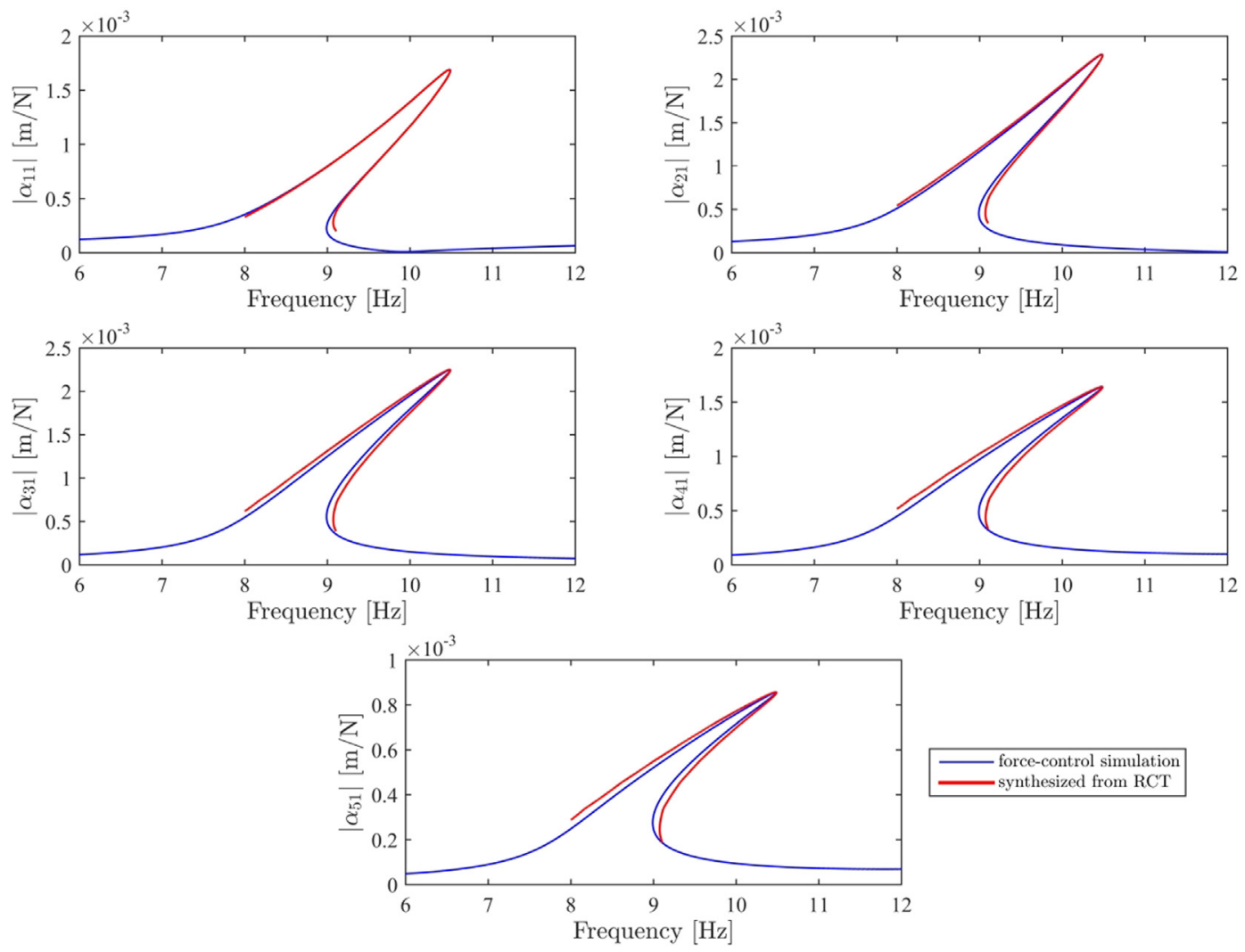

Fig. 10. Comparison of the constant-force FRFs obtained from simulated experiment, with the FRFs synthesized by using nonlinear modal parameters of the 5 DOF nonlinear system at $50 \mathrm{~N}$ forcing amplitude level. 

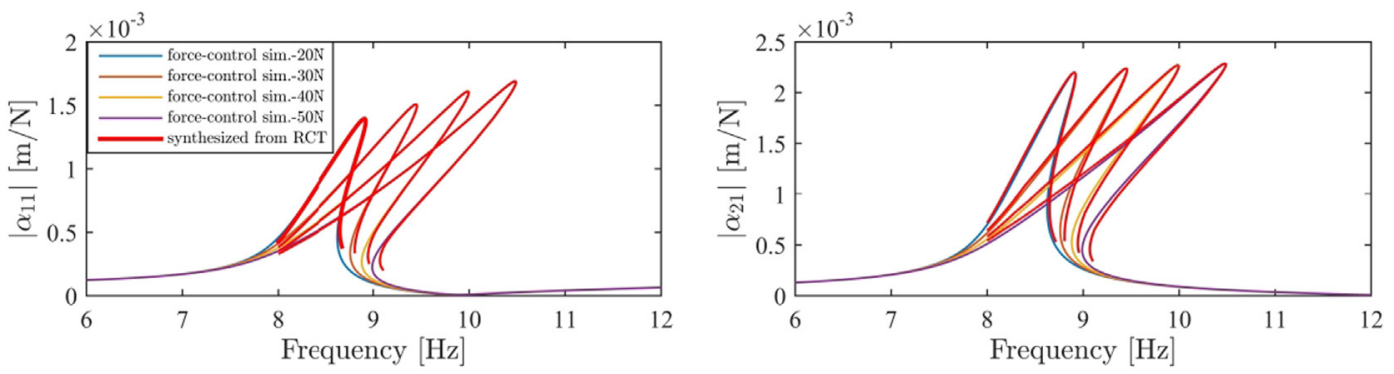

Fig. 11. Comparison of the constant-force FRFs obtained from simulated experiment, with the FRFs synthesized by using nonlinear modal parameters of the 5 DOF nonlinear system at several different forcing amplitude levels.

\subsection{Experimental extraction of constant-force FRFs by using HFS}

As an alternative to the analytical model based on identified modal parameters, untested constant-force FRFs can also be determined directly from experimental data by using the HFS concept. To do so, first of all, harmonic force spectra already obtained from RCT simulations at 10 different displacement amplitude levels, as shown in Fig. 4, were combined to construct the HFS given in Fig. 12(a). Then, the surface was cut with the $50 \mathrm{~N}$ constant-amplitude plane. The curve intersecting this plane and the HFS is nothing but the frequency response curve of the 1 st DOF corresponding to $50 \mathrm{~N}$ forcing level. Finally, the receptance curve for the 1st DOF was obtained by normalizing the frequency response curve with the amplitude of the excitation force. Comparison of the receptances obtained from the HFS approach with the ones synthesized by using identified modal parameters, as well as with the original receptances obtained from simulated force-control experiment is shown in Fig. 12(b).

\section{Experiments}

\subsection{T-beam}

The first experimental setup used to validate the proposed method is shown in Fig. 13. The test rig consists of a cantilever beam supported at its free end by two metal strips which exhibit distributed geometric nonlinearity due to large deformations. Dimensions of the rig can be found in [26]. This experimental study focuses on the frequency response around the first nonlinear mode of the structure, where a strong stiffening nonlinearity is observed.

During experiments, the system was excited with a B\&K shaker attached to its T-junction via a push-rod with a Dytran $1022 \mathrm{~V}$ force transducer as shown in Fig. 13. The vibration response was measured by using a Dytran 3225M23 miniature accelerometer attached to the top of the T-junction. All measurements and closed loop controls were accomplished by LMS SCADAS Mobile data acquisition system and LMS Test Lab. software package. The upper and lower frequency limits of the stepped-sine tests were selected based on FRF data obtained from preliminary broadband random testing. The fre-
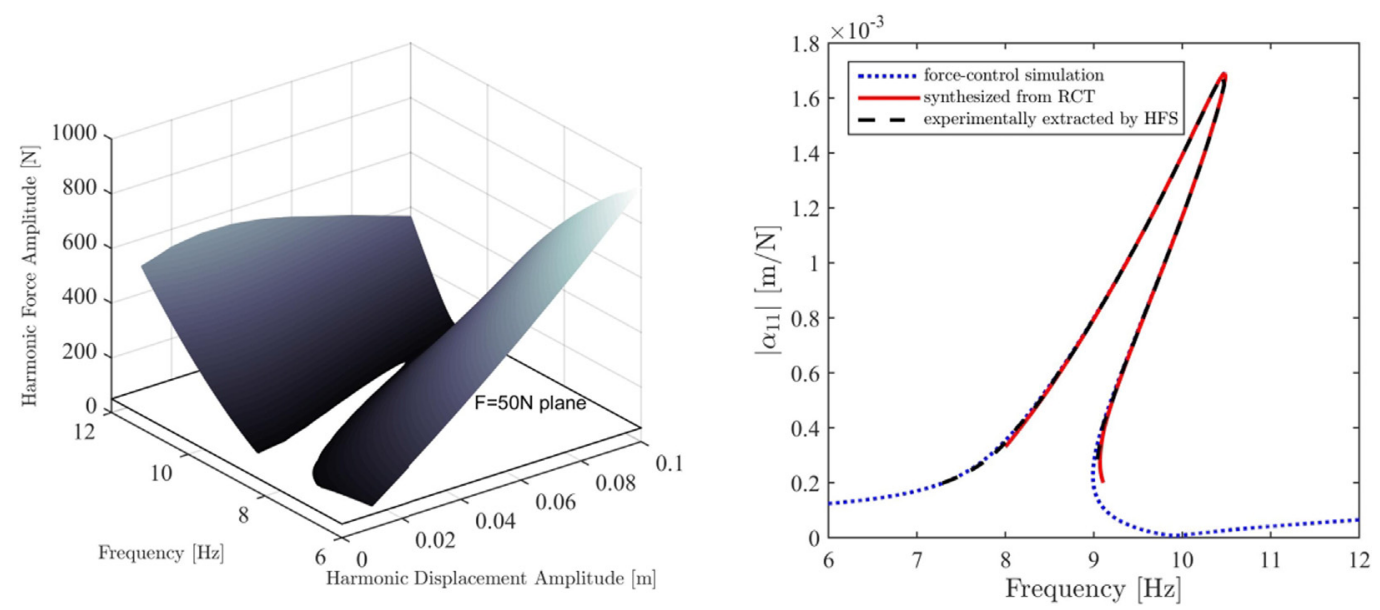

Fig. 12. (a) HFS of the 5 DOF nonlinear system (b) Comparison of the receptances extracted by using the HFS approach, with the ones synthesized by using the nonlinear modal parameters identified from RCT, and with the original receptances obtained from simulated force-control experiment at $50 \mathrm{~N}$. 

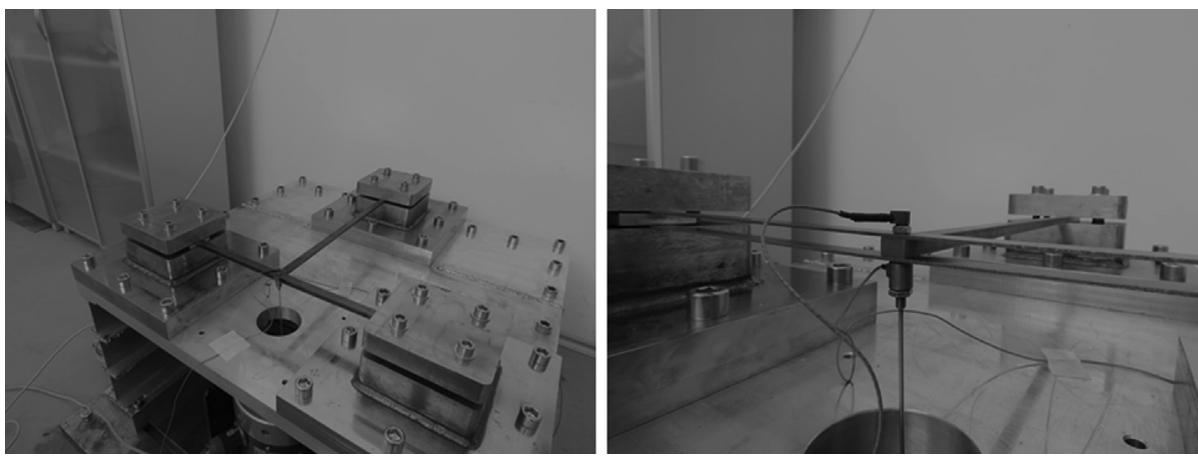

Fig. 13. The T-beam experimental setup.

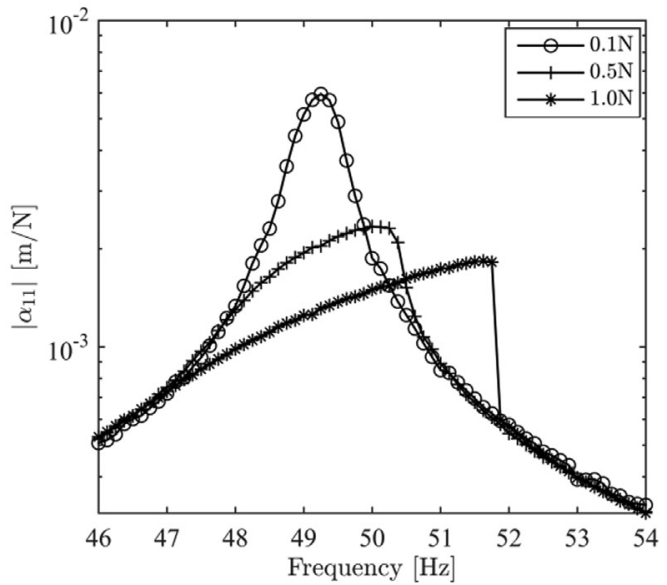

(a)

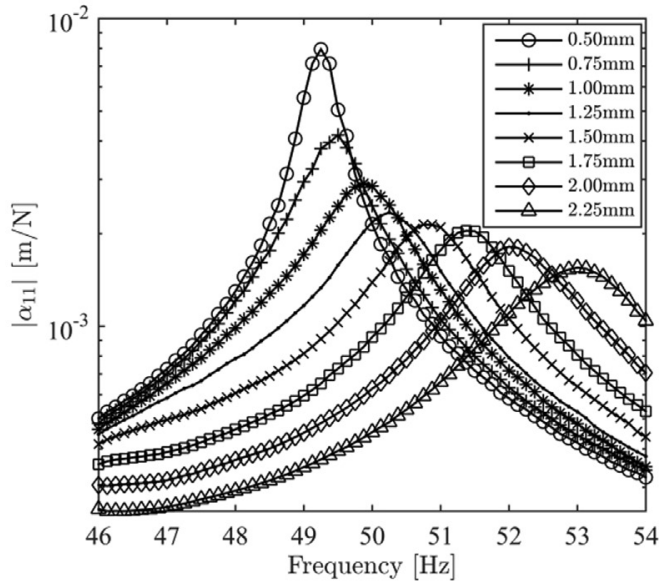

(b)

Fig. 14. (a) Constant-force FRFs of the T-beam measured by the classical force-control approach (b) constant-response FRFs of the T-beam measured by the RCT approach.

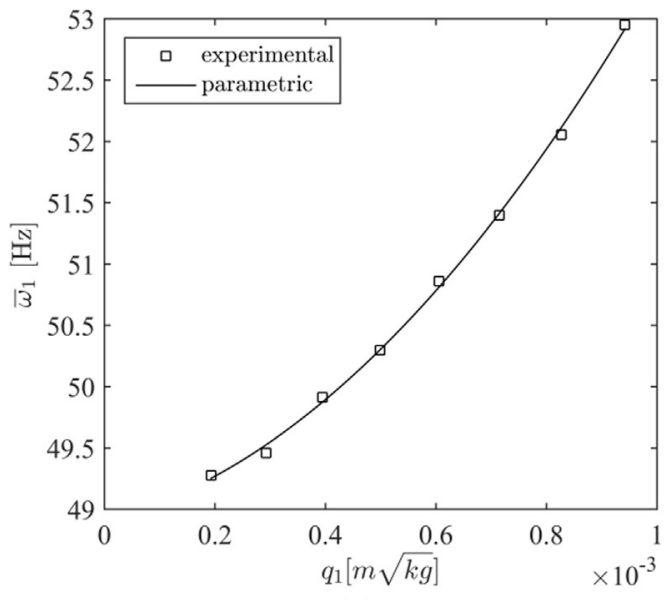

(a)

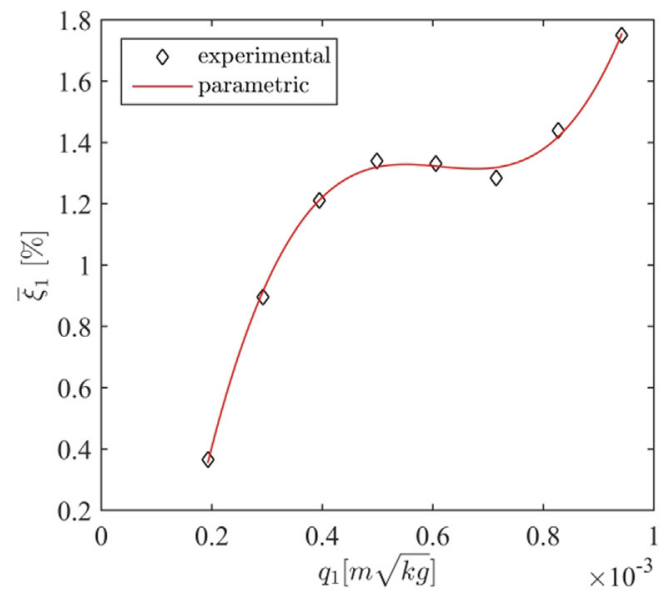

(b)

Fig. 15. Variation of the modal parameters corresponding to the 1st mode of the T-beam with modal response level (a) natural frequency (b) viscous modal damping ratio. 


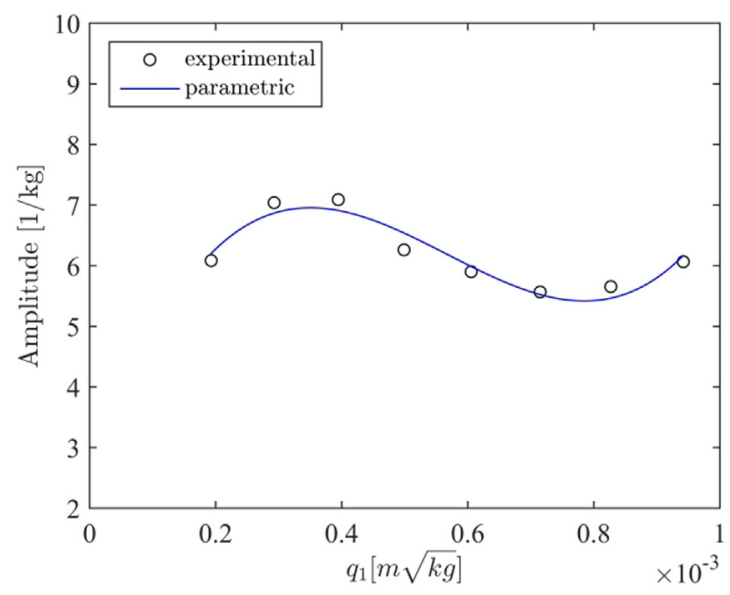

Fig. 16. Variation of the modal constant corresponding to the 1 st mode of the T-beam with modal response level.

quency step was taken to be $0.125 \mathrm{~Hz}$. After putting the control signal between the tolerance limits of the reference signal, the controller waits for a number of delay cycles to switch to the next frequency point.

First of all, a series of stepped-sine tests was conducted to characterize the nonlinearity by using classical force-control approach. Constant-force FRFs measured at 3 different excitation levels are shown in Fig. 14(a). The shift of the FRF-peak toward higher frequencies with increasing excitation level and the jump phenomenon observed at the highest level are clear indications of strong stiffening nonlinearity. Force-control tests were followed by a series of stepped-sine tests with RCT strategy. Constant-response FRFs of the T-beam measured at 8 different constant displacement amplitude levels, ranging
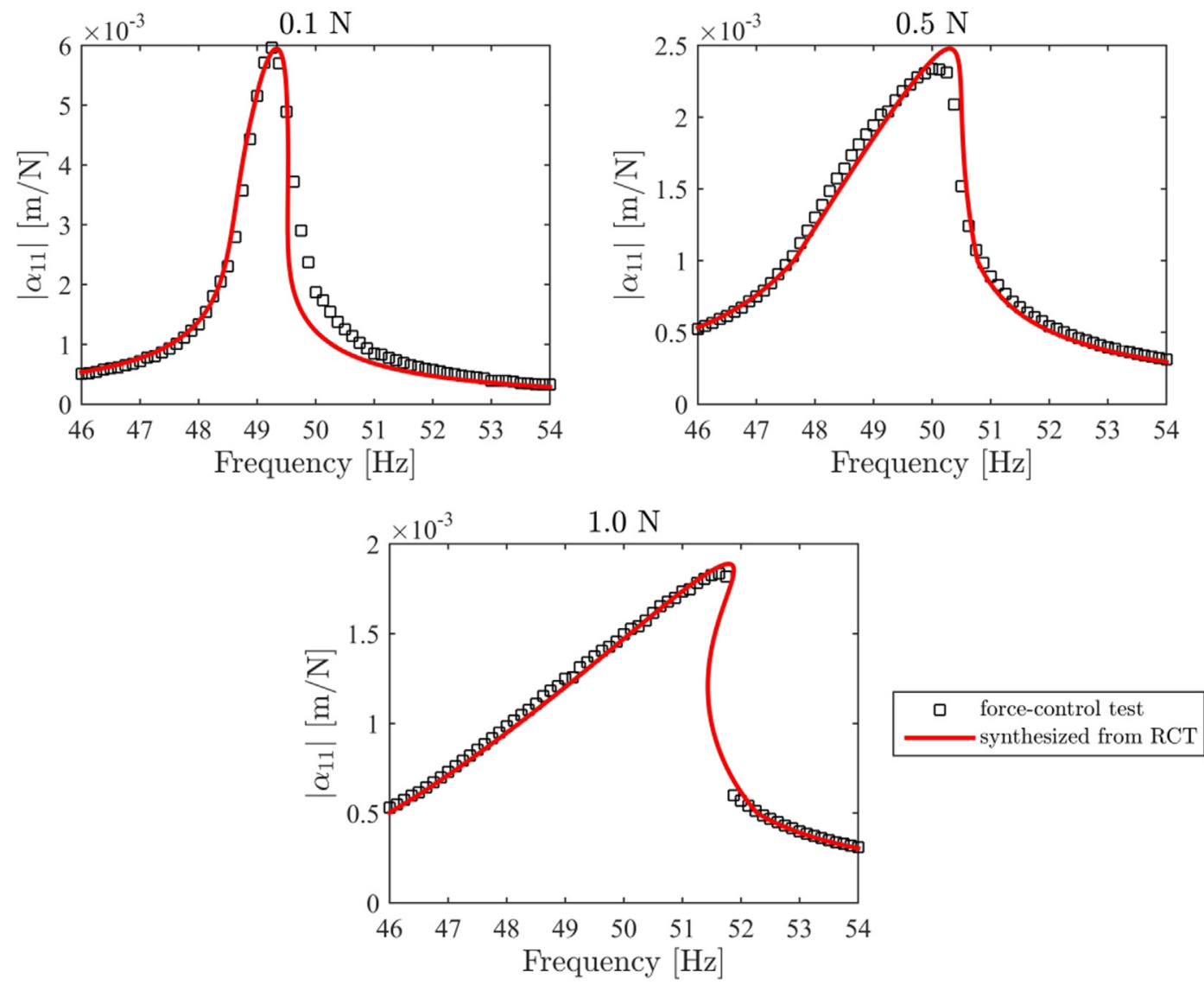

Fig. 17. Comparison of constant-force FRFs obtained from force-control test with FRFs synthesized by using nonlinear modal parameters of the T-beam. 


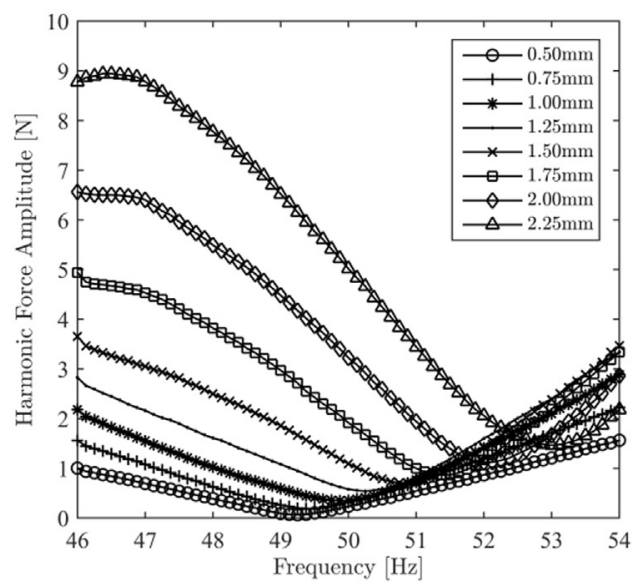

(a)

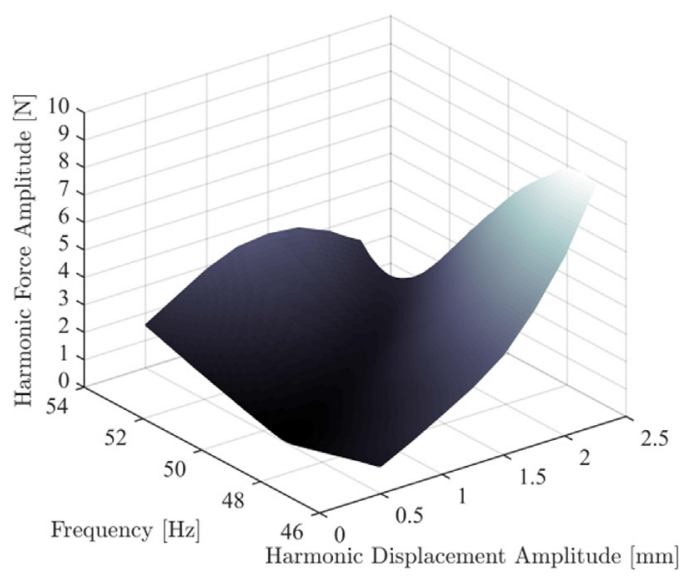

(b)

Fig. 18. (a) Harmonic force spectra of the T-beam measured during RCT (b) HFS of the T-beam constructed by collecting harmonic force spectra and using linear interpolation.

from $0.50 \mathrm{~mm}$ to $2.25 \mathrm{~mm}$, are shown in Fig. 14(b). Although the proposed method requires collecting more data compared to some other approaches, since response control is easier than force control due to more predictable (quasi-linear) behavior of the system, the response-controlled testing is much faster. For example, in the T-beam experiments, a single responsecontrolled testing is about 3 times faster than a single force-controlled testing. Therefore, total experimental effort does not increase, at least compared to force-control testing.

Fig. 15 and Fig. 16 show variations of the nonlinear modal parameters corresponding to the 1 st mode of the T-beam with respect to modal amplitude. These parameters were extracted from constant-response FRFs given in Fig. 14(b) by using linear modal analysis module of the LMS Test Lab., which is called PolyMAX. Fig. 15 and Fig. 16 show also parametric models of the nonlinear modal parameters obtained by polynomial curve fitting.

Fig. 17 compares the constant-force FRFs synthesized by employing response level dependent values of the identified modal parameters in Eq. (24), with the ones directly measured from force-controlled stepped sine testing. The agreement between the computational and experimental results is found to be quite satisfactory. Furthermore, although the forcecontrol testing cannot capture the unstable branch, it is computationally obtained by using the theoretical model based on identified modal parameters, by employing the arc-length continuation algorithm in solving Eq. (24).

Harmonic force spectra measured at different displacement amplitude levels during RCT are illustrated in Fig. 18(a). The HFS of the T-beam is constructed by collecting these force spectra together and using linear interpolation as shown in Fig. 18 (b). The frequency response curve corresponding to $1 \mathrm{~N}$ excitation force level is obtained by cutting the HFS with $1 \mathrm{~N}$ constant-amplitude plane as demonstrated in Fig. 19(a). The frequency response curve obtained from the HFS is compared

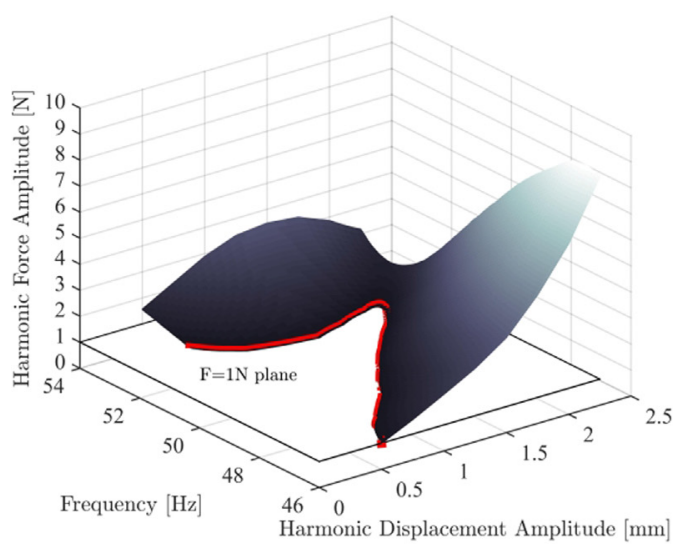

(a)

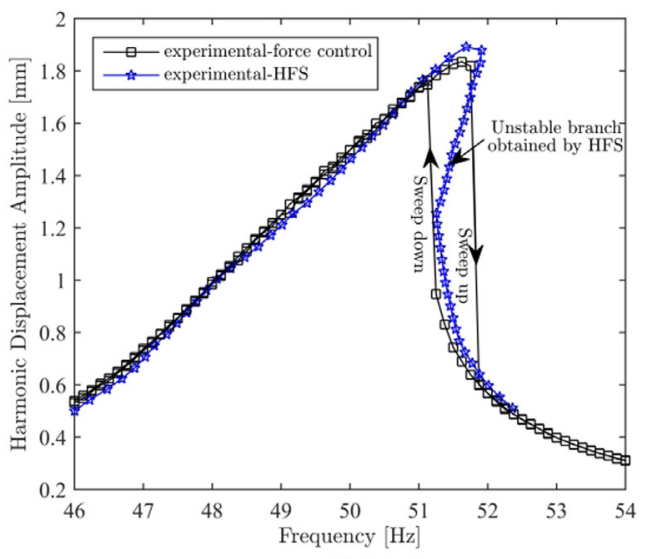

(b)

Fig. 19. (a) Determination of the harmonic response spectrum with any existing unstable branch by cutting HFS with a constant force plane (b) Comparison of the response spectrum obtained by HFS with the one obtained by force controlled testing. 


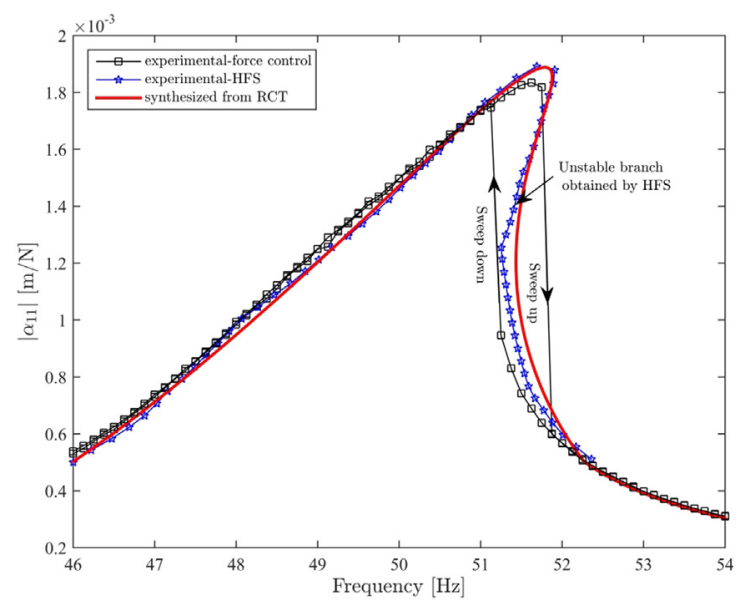

Fig. 20. Comparison of the receptances of the T-beam extracted by using the HFS approach with the ones synthesized by using nonlinear modal parameters identified from RCT, and with the original receptances obtained from force-control experiment at $1 \mathrm{~N}$.

with the one directly obtained from the force-controlled stepped-sine testing is given in Fig. 19(b). Although the HFS is capable of capturing the unstable branch completely, classical force-controlled testing can only capture it until the turning points at the cost of multiple runs with different sweep directions. The unstable branches of constant-force frequency response curves can also be determined by using the $C B C$ method, where a set of curves are obtained fixing the frequency and varying the response level. Then theses curves, which require considerable time to obtain experimentally as reported by the authors, are processed with Gaussian process regression [17].

To conclude this section, constant-force FRFs determined from the proposed method for $1 \mathrm{~N}$ excitation level are compared to the one obtained from classical force-controlled stepped-sine testing in Fig. 20. The agreement between all three approaches is found to be satisfactory.

\subsection{Real missile}

\subsubsection{Identification of nonlinear modal parameters and synthesis of constant-force FRFs}

The test setup of the real missile structure is shown in Fig. 21. Modal tests were conducted with free-free boundary conditions by using bungee-chords, lengths of which were adjusted such that rigid body mode frequencies of the missile struc-

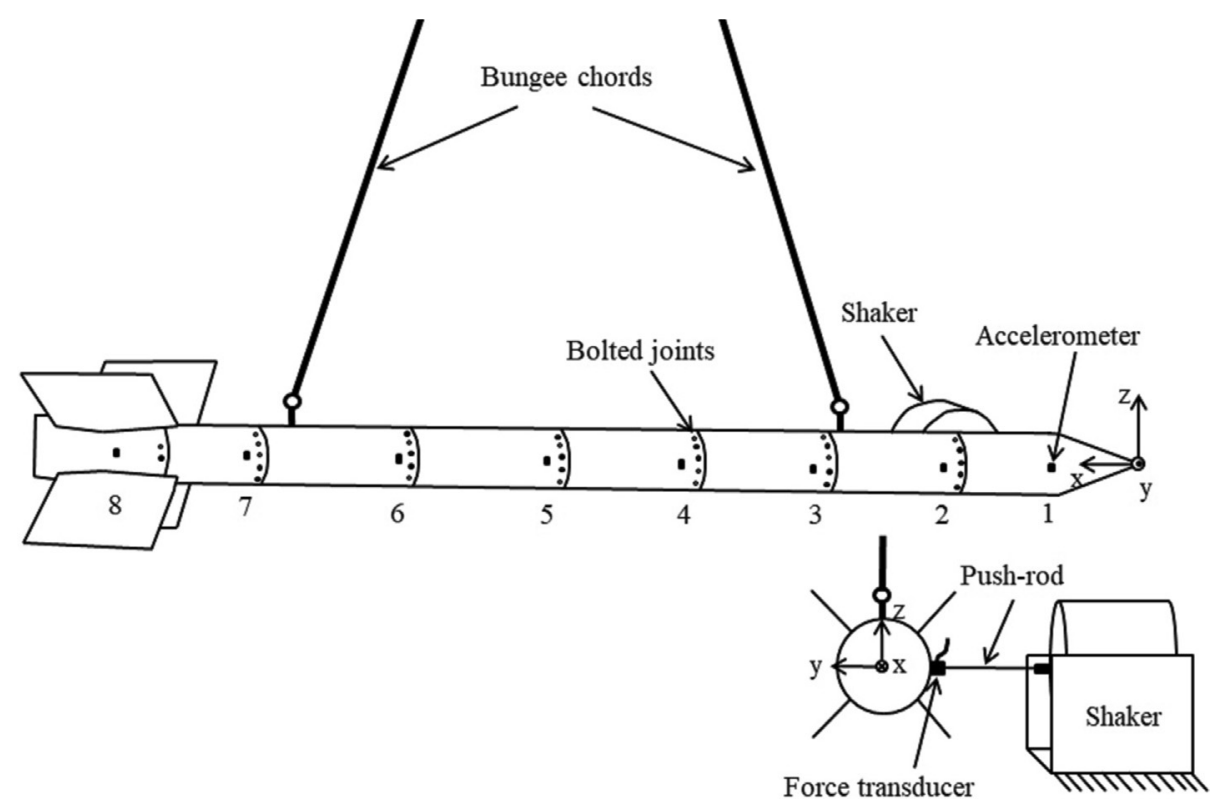

Fig. 21. Sketch of the experimental setup of the real missile. 

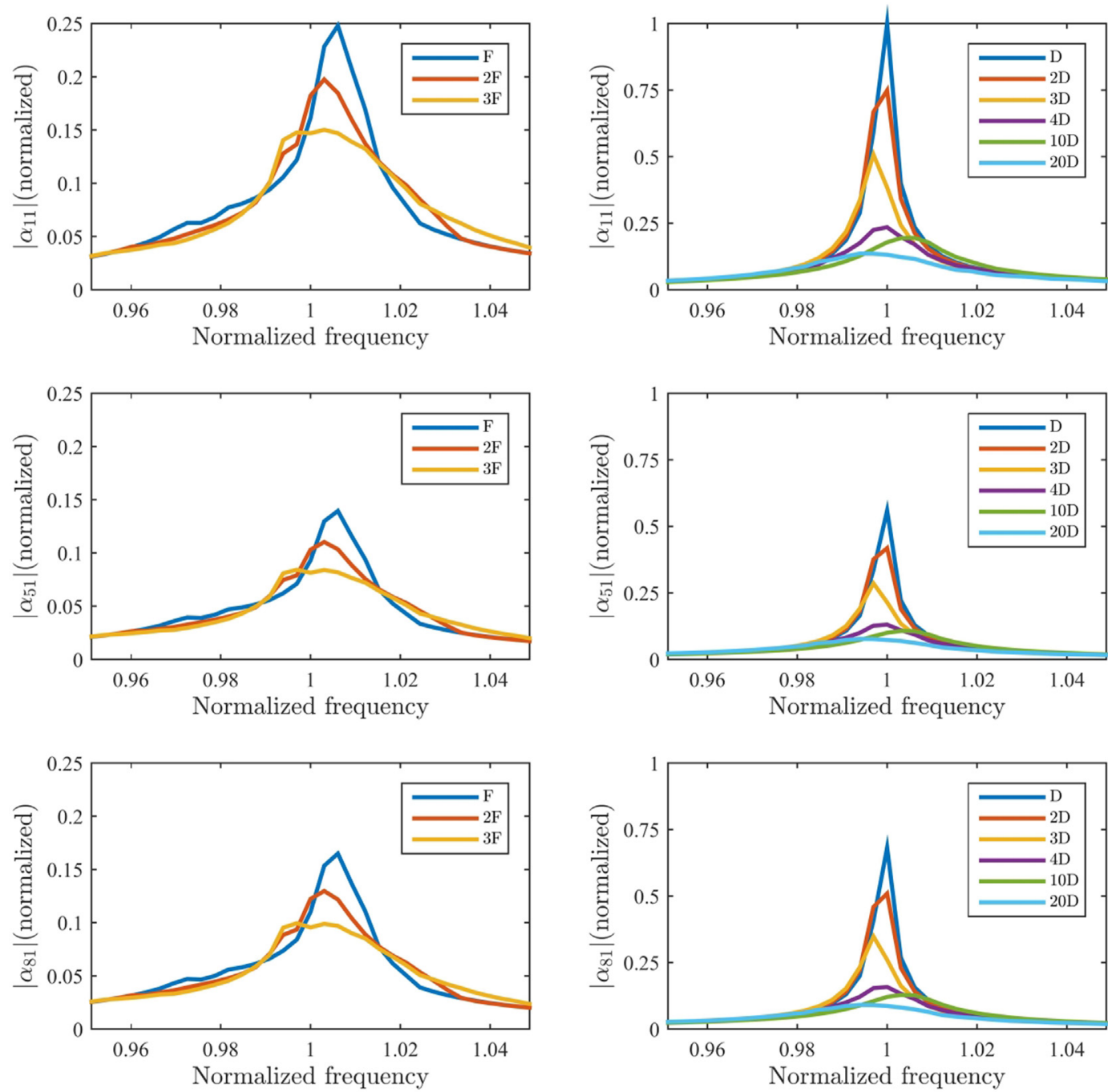

(a)

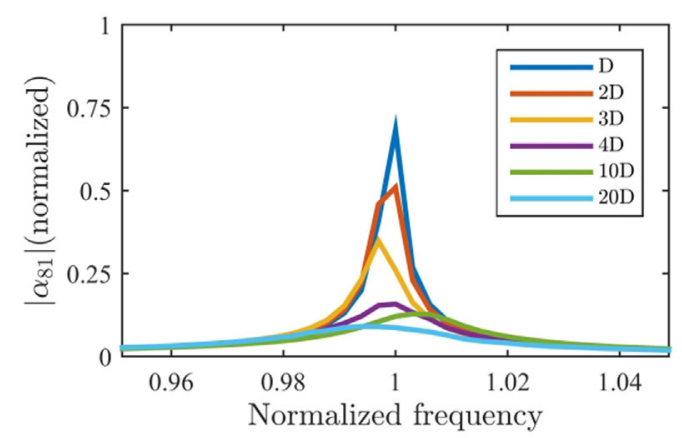

(b)

Fig. 22. (a) Constant-force FRFs of the missile structure measured by the classical force-control approach (b) constant-response FRFs of the missile structure measured by the RCT approach.

ture were kept far below its first elastic mode. In total, 8 Dytran 3225M23 miniature accelerometers were attached along the centerline of the missile and they were enumerated from 1 to 8 , as shown in Fig. 21. In this study, the focus was on the first elastic mode of the system, so the structure was excited with a single shaker in y-direction. An MB Dynamics Modal 110 shaker was attached to the structure via a push-rod with a Dytran 1051V4 force transducer at point 1 . All measurements and closed loop controls were accomplished by LMS SCADAS Mobile data acquisition system and LMS Test Lab. software package.

The locations of the bolted joints, connecting different parts of the missile are also shown in Fig. 21. All bolts are identical and have the same preload which was applied by a torque wrench during the assembly process.

The missile structure was first subjected to a series of stepped-sine tests by using classical force-control approach. The constant-force FRFs measured at three different excitation levels (F, 2F and 3F), and corresponding to three different accelerometer locations are illustrated in Fig. 22(a). The shift of the FRF-peak toward lower frequencies and the decrease in the FRF peak amplitude with increasing excitation level indicate softening nonlinear behavior with increasing nonlinear damping. The test campaign continued with a series of stepped-sine tests conducted at 14 different displacement amplitude levels (ranging from D to 20D) with RCT strategy. For the sake of readability, constant-response FRFs corresponding to 6 selected displacement amplitude levels are illustrated in Fig. 22(b).

Variation of modal parameters identified from constant-response FRFs are shown in Fig. 23 and Fig. 24. Confidence intervals were obtained by repeating each test several times during the test campaign. The first important observation made from 


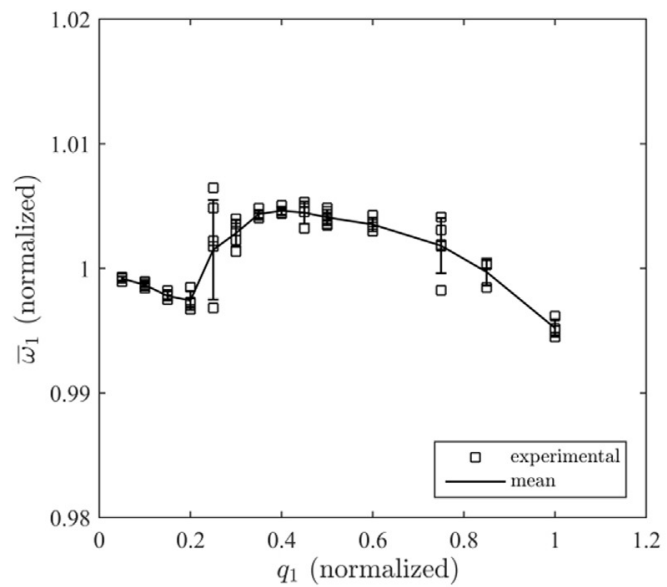

(a)

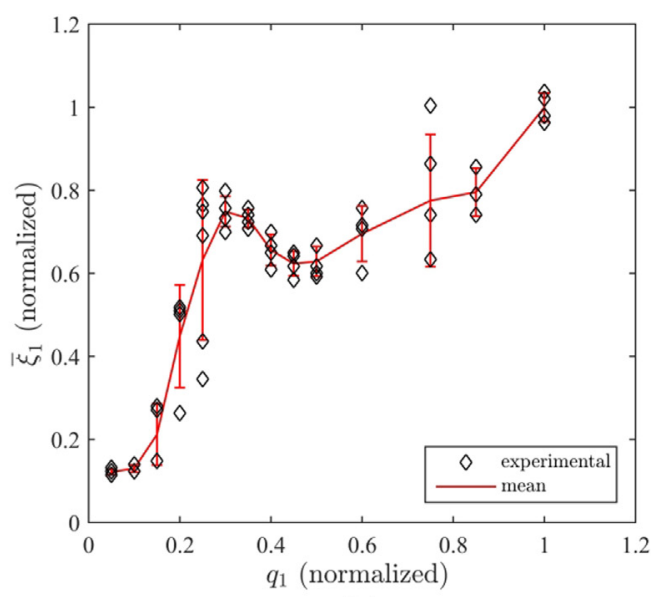

(b)

Fig. 23. Variation of the modal parameters corresponding to the 1st mode of the missile structure with modal response level (a) natural frequency (b) viscous modal damping ratio.

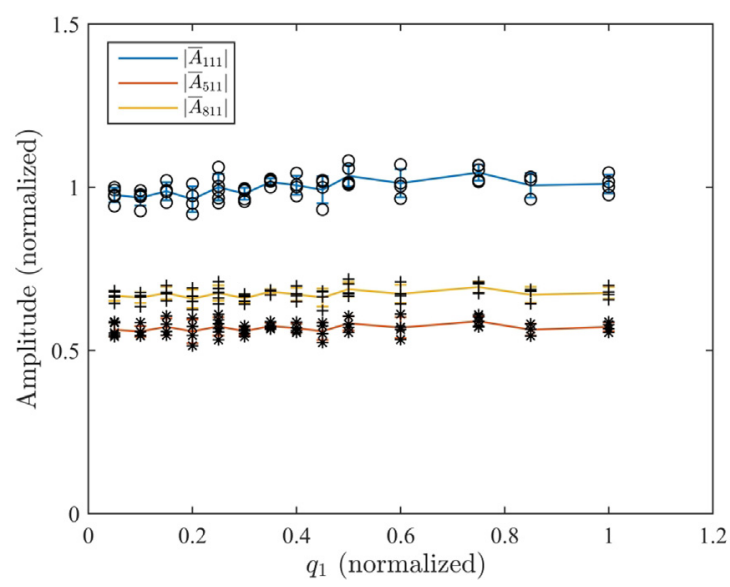

Fig. 24. Variation of modal constants corresponding to the 1st mode of the missile structure with modal response level.

Fig. 23 is the order of magnitude change in the nonlinear modal damping ratio and a very small change in the natural frequency, which indicate that the system exhibits considerable damping nonlinearity but weak stiffness nonlinearity. Another observation worth mentioning is the transition region (between 0.2 and 0.4 ), where nominal damping increases drastically,

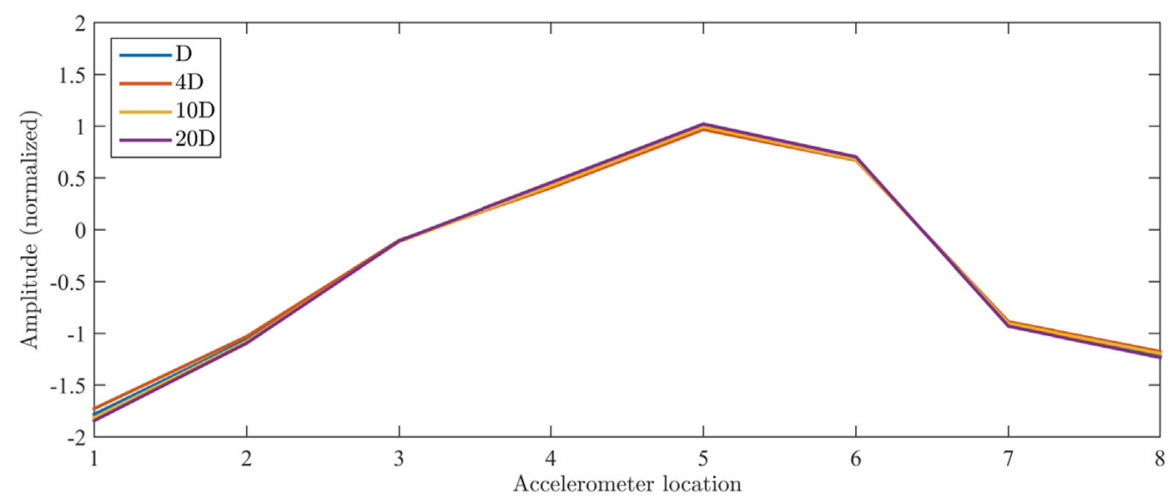

Fig. 25. 1st NNM of the missile structure experimentally extracted by using the modal constants identified from RCT. 

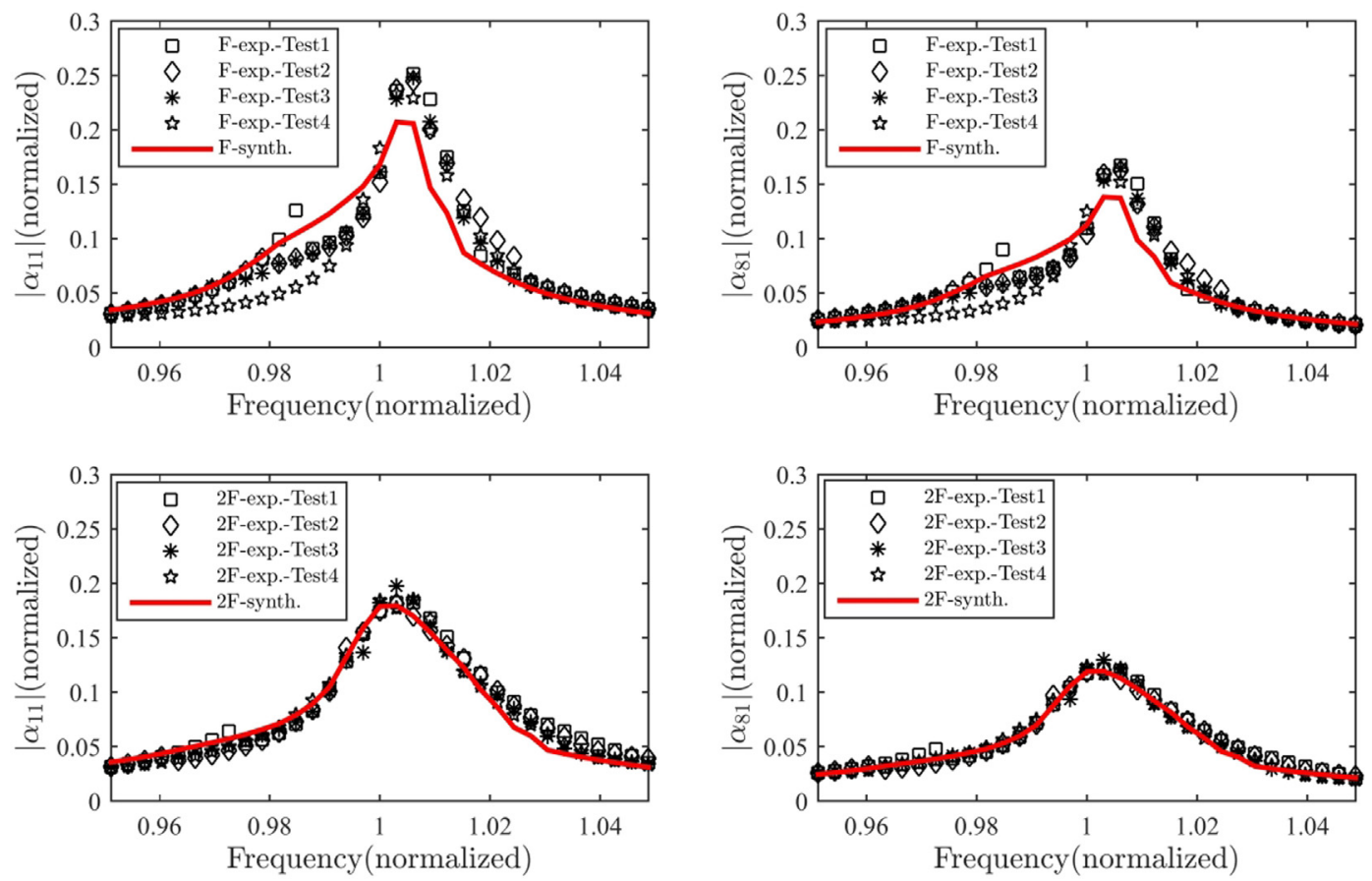

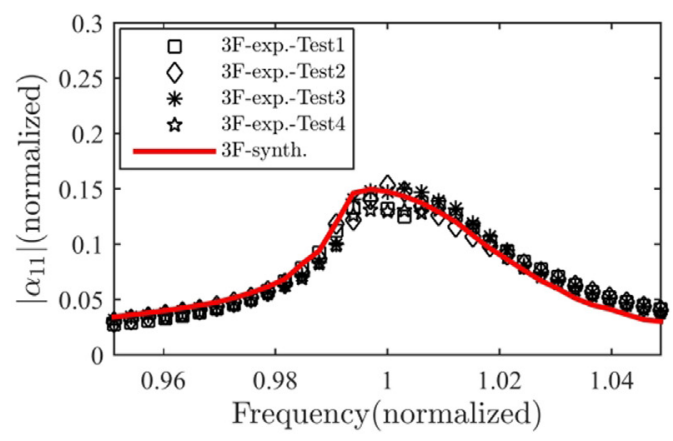

(a)

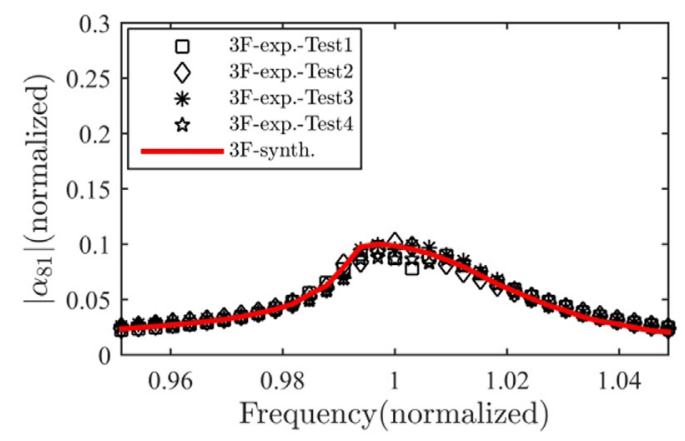

(b)

Fig. 26. Comparison of the constant-force FRFs obtained from force-controlled test, with FRFs synthesized by using nonlinear modal parameters of the missile: (a) driving point FRF (point1 in Fig. 21) (b) FRF between tail point (point 8 in Fig. 21) and driving point.

and the considerably large confidence intervals in this region. This transition region may be an indication of the stick to slip transition (slipping means more friction and damping). On the other hand, large confidence intervals may result from combined effects of several different environmental conditions such as temperature change and changes of contact conditions due to vibration at the joint interfaces.

Due to the negligible change in the identified modal constants with response level, as illustrated in Fig. 24, it can be concluded that the shape changes in the NNM with vibration response level are also negligible, as can be seen in Fig. 25.

This section is concluded with the comparison of the constant-force FRFs synthesized by employing response level dependent values of the identified modal parameter (see Fig. 23 and Fig. 24) in Eq. (24), with the ones directly measured from force-controlled stepped sine testing, which is shown in Fig. 26. The agreement between the computational and experimental results is found to be quite satisfactory at moderate and high excitation levels (i.e. at $2 \mathrm{~F}$ and $3 \mathrm{~F}$ ). At the lowest excitation level, repeatability of the measured constant-force FRFs is poor, which is the cause of large confidence intervals observed in the nonlinear damping ratio shown in Fig. 23(b). Consequently, the agreement between the computational and experimental FRFs at the lowest excitation level is not as good as that obtained at moderate and high excitation levels.

\subsubsection{Effect of bolt preload on modal parameters}

The modal parameters given in Fig. 23 were obtained for the bolt tightening torque of T1 which is the design torque level of the bolts shown in Fig. 21. In order to determine the effects of bolt preload on the modal parameters, RCTs were repeated 


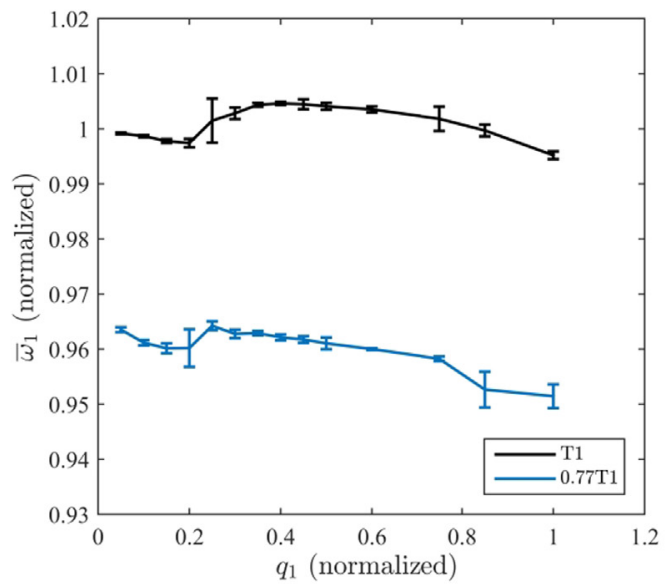

(a)

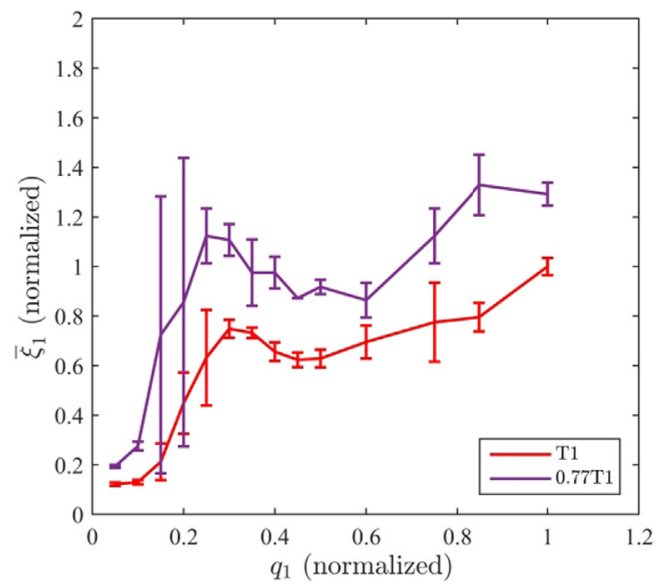

(b)

Fig. 27. Effect of bolt preload on the modal parameters (a) natural frequency (b) viscous modal damping ratio.

for a lower torque level of $0.77 \mathrm{~T} 1$. The modal parameters obtained at these two different torque levels are compared in Fig. 27.

It can be observed from Fig. 27(a) that when the bolt preload is reduced, the natural frequency decreases, i.e. the structure becomes more flexible. Furthermore, since less preload means more sliding (i.e. more friction and damping), nonlinear damping at the joints is enhanced when the preload is reduced as shown in Fig. 27(b). Another interesting observation relevant to Fig. 27 is that the percentage increase in the modal damping ratio exceeds $50 \%$ at various displacement levels, while the decrease in the natural frequency is limited to 3-5\%. Therefore, it is concluded that modal damping, compared to natural frequency, is much more sensitive to bolt preload. In other words, stiffness is affected much less than damping. To know the amount of increase that can be achieved in modal damping by decreasing bold preload may be an important parameter in designing such structures from an aeroelastic perspective.

\section{Conclusions}

This paper proposes a nonlinear experimental modal analysis approach based on response-controlled stepped sine testing (RCT). The proposed approach is applicable to systems with several nonlinearities at various different locations, provided that modes are well separated and no internal resonances occur. In this approach, a series of modal tests is conducted by using RCT strategy. In each test, the displacement amplitude of the driving point is kept at a constant level, as a result of which measured constant-response FRFs come out in quasi-linear form. Subsequently, by applying standard linear modal analysis techniques to these constant-response FRFs, modal parameters corresponding to a specific nonlinear mode are experimentally extracted as functions of the modal amplitude. The identified response dependent modal parameters are used to synthesize near-resonant frequency response curves, even with unstable branches if there is any, for unmeasured harmonic forcing cases without any extra experimental effort. As the experimentally extracted nonlinear modal parameters are response level dependent, the computation of frequency response curves require an iterative solution. In this work, Newton's Methods and arc-length continuation algorithm is used which makes it possible to calculate any unstable branch as well. The proposed approach provides also an alternative way of determining frequency response curves (once again without any extra experimental effort) by directly extracting isocurves of constant-amplitude forcing from the Harmonic Force Surface measured during RCT, which is a novel concept proposed in this paper. Furthermore, by using multiple sensors during RCT, mass normalized NNMs can also be experimentally extracted from the identified modal constants.

Considering state-of-the-art techniques, the proposed method has several contributions. Firstly, it relies on standard controllers (available in commercial modal testing hardware and driven by commercial software), which makes it very attractive especially for industrial applications. Secondly, identification of modal damping and mass normalization of NNMs is straightforward with the proposed method, by applying linear modal analysis methods available in commercial software packages to constant-response FRFs measured during RCT. Finally, in case of strongly nonlinear systems, the method is capable of capturing unstable branches of frequency response curves either computationally by using identified modal parameters or experimentally by using the HFS concept. However, it should be noted that the proposed method is limited to systems which have well separated modes and which do not exhibit internal resonances within the energy range of interest. An investigation on the applicability of the proposed method to the case of internal resonances is in our future work program.

In this study, the application and validation of the approach proposed are demonstrated on numerical and experimental case studies. In the numerical example, the method is successfully applied to a 5 DOF lumped system with strong conservative nonlinearity. A very good match is obtained between the computational results and the simulated experimental 
results, even for high forcing cases where very strong nonlinear effects with unstable branches are observed. Next, the method is validated on a cantilever beam supported at its free end by thin metal strips which exhibit geometric nonlinearity due to large deformations. Frequency response curves for constant amplitude harmonic forcing are accurately predicted (including the unstable branch which appears at the highest excitation level due to strong stiffening nonlinearity) both computationally from the identified nonlinear modal parameters, and experimentally from the HFS whose prominent feature is the experimental extraction of the unstable branches together with accurate determination of the turning points of frequency response curves at constant excitation level. Finally, the method is applied to a real missile structure which exhibit moderate damping nonlinearity. In this case study, standard force-controlled modal test approach leads to nonlinear FRFs, and linear modal analysis fails to predict even an approximate value for structural damping. However, by using the measured constant-response FRFs, the proposed method accurately predicts modal damping ratio as a function of modal amplitude, which has an important role in the accuracy of aeroelastic analyses. Missile example also shows that bolt preload has a considerable effect on the nonlinear modal damping, which makes it an important design parameter from an aeroelastic perspective.

\section{CRediT authorship contribution statement}

Taylan Karaağaçlı: Conceptualization, Methodology, Software, Validation, Formal analysis, Investigation, Writing - original draft. H. Nevzat Özgüven: Supervision, Conceptualization, Methodology, Writing - review \& editing.

\section{Declaration of Competing Interest}

The authors declare that they have no known competing financial interests or personal relationships that could have appeared to influence the work reported in this paper.

\section{Acknowledgment}

The provision of TÜBITAK-SAGE for modal testing and analysis capabilities is gratefully acknowledged.

\section{References}

[1] G. Kerschen, K. Worden, A.F. Vakakis, J.C. Golinval, Past, present and future of nonlinear system identification in structural dynamics, Mech. Syst. Sig. Process. 20 (2006) 505-592.

[2] J.P. Noël, G. Kerschen, Nonlinear system identification in structural dynamics: 10 more years of progress, Mech. Syst. Signal Process. 83 (2017) 2-35.

[3] R.M. Rosenberg, The normal modes of nonlinear n-degree-of-freedom systems, J. Appl. Mech. 29 (1962) 7-14.

[4] R.M. Rosenberg, On nonlinear vibrations of systems with many degrees of freedom, Adv. Appl. Mech. 9 (1966) 155-242.

[5] W. Szemplinska-Stupnicka, The modified single mode method in the investigations of the resonant vibrations of nonlinear systems, J. Sound Vib. 63 (4) (1979) 475-489.

[6] S. Setio, H.D. Setio, L. Jezequel, A method of nonlinear modal identification from frequency response tests, J. Sound Vib. 158 (3) (1992) 497-515.

[7] C. Gibert, Fitting measured frequency response using nonlinear modes, Mech. Syst. Sig. Process. 17 (1) (2003) 211-218.

[8] F. Thouverez, Presentation of the ECL benchmark, Mech. Syst. Sig. Process. 17 (1) (2003) 195-202.

[9] D. Göge, U. Füllekrug, M. Sinapius, M. Link, L. Gaul, Advanced test strategy for identification and characterization of nonlinearities of aerospace structures, AIAA J. 43 (5) (2005) 974-986.

[10] S.F. Masri, T.K. Caughey, A nonparametric identification technique for nonlinear dynamic problems, J. Appl. Mech. 46 (1979) $433-447$.

[11] M.F. Platten, J.R. Wright, G. Dimitriadis, J.E. Cooper, Identification of multi-degree of freedom nonlinear systems using an extended modal space model, Mech. Syst. Sig. Process. 23 (1) (2009) 8-29.

[12] M.F. Platten, J.R. Wright, J.E. Cooper, G. Dimitriadis, Identification of a nonlinear wing structure using an extended modal model, AIAA J. Aircraft 46 (5) (2009) 1614-1626.

[13] U. Fuellekrug, D. Goege, Identification of weak nonlinearities within complex aerospace structures, Aerosp. Sci. Technol. 23 (2012) 53-62.

[14] M. Peeters, G. Kerschen, J.C. Golinval, Dynamic testing of nonlinear vibrating structures using nonlinear normal modes, J. Sound Vib. 330 (2011) $486-$ 509.

[15] M. Peeters, G. Kerschen, J.C. Golinval, Modal testing of nonlinear vibrating structures based on nonlinear normal modes: experimental demonstration, Mech. Syst. Sig. Process. 25 (2011) 1227-1247.

[16] S. Peter, R.I. Leine, Excitation power quantities in phase resonance testing of nonlinear systems with phase-locked-loop excitation, Mech. Syst. Sig. Process. 96 (2017) 139-158.

[17] L. Renson, A. Gonzalez-Buelga, D.A.W. Barton, S.A. Neild, Robust identification of backbone curves using control-based continuation, J. Sound Vib. 367 (2016) $145-158$.

[18] V. Denis, M. Jossic, C. Giraud-Audine, B. Chomette, A. Renault, O. Thomas, Identification of nonlinear modes using phase-locked-loop experimental continuation and normal form, Mech. Syst. Sig. Process. 106 (2018) 430-452.

[19] S. Peter, M. Scheel, M. Krack, R.I. Leine, Synthesis of nonlinear frequency responses with experimentally extracted nonlinear modes, Mech. Syst. Sig. Process. 101 (2018) 498-515.

[20] M. Scheel, S. Peter, R.I. Leine, M. Krack, A phase resonance approach for modal testing of structures with nonlinear dissipation, J. Sound Vib. 435 (2018) 56-73.

[21] Ö. Tanrıkulu, B. Kuran, H.N. Özgüven, M. İmregün, Forced harmonic response analysis of nonlinear structures using describing functions, AIAA J. 31 (7) (1993) 1313-1320.

[22] Arslan Ö., Özgüven, H.N., Modal identification of nonlinear structures and the use of modal model in structural dynamic analysis, in: Proceedings of the 26th International Modal Analysis Conference (IMAC), Orlando, USA, 2008.

[23] Ferreira J.V. , Ewins D.J., Algebraic nonlinear impedance equation using multi-harmonic describing function, 15th , Int. Modal Anal. Conf. (1997) 1595.

[24] G. Kerschen, M. Peeters, J.C. Golinval, A.F. Vakakis, Nonlinear normal modes, part I: a useful framework for the structural dynamicist, Mech. Syst. Sig. Process. 23 (1) (2009) 170-194. 
[25] S. Schwarz, L. Kohlmann, A. Hartung, J. Gross, M. Scheel, M. Krack, Validation of a turbine blade component test with frictional contacts by phaselocked-loop and force-controlled measurements, J. Eng. Gas Turbines Power 142 (5) (2020), https://doi.org/10.1115/1.4044772.

[26] Ö. Arslan, M. Aykan, H.N. Özgüven, Parametric identification of structural nonlinearities from measured frequency response data, Mech. Syst. Signal Process. 25 (2011) 1112-1125. 